\title{
Determinantes socioeconómicos de migración en México*
}

\author{
James B. Pick, ${ }^{* *}$ Glenda L. Tellis, ${ }^{* * *}$ Edgar W. Butler*** \\ y Suhas Pavgi****
}

En este estudio se examina la influencia socioeconómica en la frecuencia de migración y movimientos transitorios en cuatro regiones de México. El análisis se basa en los datos colectados en la Encuesta Mexicana de Fecundidad 1976-1977, y considera la influencia de: educación, ocupación, alfabetización, lugar de residencia y fecundidad. Los métodos utilizados para el análisis son regresión y regresión logística. En la regresión logística las tres primeras transiciones de movimientos se separaron y se analizaron individualmente. Los resultados muestran una sucesión de influencias socioeconómicas dependiendo del estado de transición del movimiento. Para la transición 0-1 + el mayor efecto es el lugar de residencia; para la transición de 1-2 + es la ocupación de migrante; y para la transición de $2-3+$ los efectos inconsistentes de edad y educación. Se muestran los efectos específicos de acuerdo con la edad y la región.

\section{Introducción}

El crecimiento de la población mexicana ha sido muy rápido desde 1940. Se calcula que la población en 1988 era de 83.5 millones, mientras que en 1980 la población fue de 67.4 millones. Estos cálculos, los cuales asumen un decremento en las tasas de fecundidad, estiman que para el año 2000 la población será de $104.5 \mathrm{mi}$ llones (Population Reference Bureau, 1988).

En las dos décadas pasadas, el rápido crecimiento de la pobla-

* La elaboración del proyecto de investigación que aquí se presenta fue posible gracias al apoyo económico del Programa UC-MEXUS, Senado Académico de UCR, el Grupo de Investigación y Entrenamiento Colaborativo UCR-México que facilitó el desarrollo del proyecto Base de Datos UCR México. Extendemos nuestros agradecimientos a los profesores Adalberto Aguirre y Robert Singer de la UCR, así como Bill Vanore, Alex Ramírez y Larry Sautter de Comunicaciones y Centro de Cómputo, UCR, y a Juan Jiménez. Agradecemos los valiosos comentarios y sugerencias de Tonya Schuster de la Universidad de California Sur y de Diana Bustamante de la Universidad de Nuevo México.

** Graduate Schooll of Management University of California, Riverside, California, EU.

*** Department of Sociology University of California, Riverside, California, EU. **** CIBER, Inc., Dallas, Texas, EU. 
ción de México ha sido acompañado con otros grandes problemas, tales como la migración internacional hacia Estados Unidos, la rápida urbanización y patrones persistentes de migración interna dentro de México y a las regiones fronterizas con Estados Unidos (Winnie, 1981; Fukurai et al., 1987, a,b; Butler et al., 1987). Tanto la administración del presidente Miguel De la Madrid, como la de su sucesor Carlos Salinas de Gortari, han reconocido las implicaciones perjudiciales de estas tendencias para México. La administración de De la Madrid recomendó fuertemente el control de la fecundidad y adoptó una política oficial de descentralización de la población de las ciudades, especialmente de la ciudad de México.

En las siguientes décadas, el entendimiento de los patrones de migración interna será de vital importancia para lograr un balance en el reparto de recursos y el bienestar de los ciudadanos. El presente estudio analiza las influencias anteriores sobre la migración interna en cuatro regiones de México. Este estudio contribuye al conocimiento básico necesario para entender y desarrollar un plan de crecimiento y redistribución de la población.

El estudio tiene los siguientes objetivos:

1. Investigar los efectos de ciertas variables socioeconómicas seleccionadas sobre cuatro variables dependientes de la migración, una de éstas indicando la frecuencia de migración y las tres restantes representando las transiciones de movimientos para cuatro regiones de México. tantes.

2. Interpretar las causas sobresalientes de los efectos impor-

3. Evaluar, de manera particular, si existen influencias de la fecundidad sobre las variables dependientes de la migración y determinar si los efectos varían de acuerdo con las regiones.

4. Determinar si hay similitudes o diferencias en las influencias socioeconómicas sobre la migración entre dos regiones de emigrantes y dos regiones de inmigrantes históricamente importantes por sus movimientos migratorios.

5. Desarrollar generalizaciones sobre migración interna en México.

\section{Patrones de migración internos en México}

A principios de este siglo, algunos factores incrementaron las tasas de migración interna en México, incluyendo movimientos geográficos relacionados con la ruptura del sistema de haciendas y al inicio de la Revolución en 1910, avances en la tecnología del 
transporte a principios de 1900 , incremento en la información sobre oportunidades de trabajo y cambios en la economía mexicana. Los patrones de migración interna en México son muy variables. El estudio hecho por Whetten (1956) sobre migración permanente entre estados mexicanos, basados en los datos del censo de 1950 , demostró que $12.9 \%$ de la población mexicana en 1950 vivió en un estado diferente en el que nació (por ejemplo, inmigración de por vida). Las entidades federales con mayor migración de por vida fueron Baja California ( $63.4 \%$ nacidos en otros estados) y el Distrito Federal (46\%). En contraste, los estados centrales y de la región sur del Pacífico tuvieron tasas de inmigración muy bajas, en realidad hubo pérdidas netas en la migración de por vida. El estudio también muestra un incremento desproporcionadamente grande de migración neta en los estados urbanos. Los flujos migratorios más grandes se dieron entre estados contiguos, mientras que para estados no contiguos los flujos migratorios mayores fueron hacia el Distrito Federal.

La redistribución interna de la población mexicana entre 1950 y 1970 siguió un patrón similar (Winnie, 1981). En los años setenta las áreas con un crecimiento significativo fueron la megalópolis de la ciudad de México consistiendo en el Distrito Federal y el Estado de México, seguida por la región del Pacífico norte, especialmente el estado de Sinaloa. En 1980, las tres áreas metropolitanas más grandes fueron la ciudad de México, Guadalajara, en el estado de Jalisco, y Monterrey, en Nuevo León. En estas ciudades se encontraban $27.4 \%$ de la población de México (Secretaría de Programación y Presupuesto, 1984). Los primeros estudios que examinaron los componentes de migración hacia estas importantes ciudades de México generalmente se enfocaron en aspectos socioeconómicos. Los inmigrantes a la ciudad de México tendían a tener niveles de ocupación y educación más bajos que los oriundos de ésta y las mujeres inmigrantes tenían niveles sustancialmente más bajos en estas características comparándolas con los hombres inmigrantes (Muñoz et al., 1971, 1972, 1973).

Browning y Feindt (1968, 1969, 1970) en las investigaciones que hicieron durante 10 años para tres grupos sucesivos de inmigrantes a Monterrey y su integración después de su llegada, concluỷen lo siguiente:

1. Generalmente las características educacionales y ocupacionales de los inmigrantes a Monterrey tienden a ser más altas que las de la población de origen.

2. Los inmigrantes mostraron ser menos selectivos a través del tiempo. 
3. El decremento en selectividad implica un cambio en migración por grupos; por ejemplo, un patrón en el cual los inmigrantes han tenido niveles de educación y ocupación más bajos, y, probablemente, más eran casados y con familia.

4. Junto con el decremento en selectividad hubo un cambio a inmigrantes de áreas rurales y estados de origen menos desarrollados.

5. El trabajo fue el factor más importante para mudarse a Monterrey.

6. Una proporción muy alta de inmigrantes se satisfizo debido, en gran parte, al apoyo de parentesco que recibieron a su llegada.

Otras investigaciones se han enfocado con variables independientes tales como la distancia de carreteras entre dos estados, salario mensual medio, desempleo y población del estado; para las últimas tres variables se consideraron ambos origen y destino (Greenwood y Ladman, 1977, 1978, 1981). Los resultados revelan que la distancia ha tenido un efecto negativo en la migración. Para las otras variables, los resultados son más significativos de acuerdo con el punto de destino más que con el de origen. En la elección del destino el ingreso y el tamaño de la población fueron factores positivos, mientras que el desempleo fue un factor negativo. Los efectos de acuerdo con el origen de los migrantes fueron menos consistentes y menos significativos (véase también Cole y Sanders, 1983).

Existe un estudio más reciente enfocado en la inmigración a Baja California, utilizando los datos de los censos mexicanos 1900 a 1989 (Butler et al., 1987). En este trabajo al examinar diferentes mecanismos de predicción se encontró que la influencia dominante para la migración en 1900,1950, 1960, 1970 y 1980 fue la distancia entre el estado de origen y Baja California. Las variables socioeconómicas fueron únicamente importantes en 1980 y sus efectos fueron densidad de población $(-)$, alfabetización $(+)$, actividad económica $(+)$ y actividad agrícola $(+)$.

El estudio de los 992 flujos migratorios interestatales durante 1979 a 1980, incorporando organizaciones de negocios, mercado de trabajo e ingreso tanto en el estado de origen como en el estado de destino, así como también proximidad, apoyan un modelo de segmentación económica, en el cual los aspectos más importantes, además de la distancia, son la organización de los negocios y las características del mercado de trabajo (Fukurai et al. 1987a).

Los flujos migratorios en México también han seguido patrones a largo plazo. Por ejemplo, el flujo migratorio hacia Baja California fue muy consistente entre 1900 y 1980 . En términos porcentuales, en 1980 y 1970, los inmigrantes de por vida en Baja 
California provienen principalmente de Jalisco $(19.9,22.8)$, Sonora $(12.0,11.8)$ y Sinaloa $(11.8,10.7)$. Aunque no hay datos específicos disponibles para Baja California, aparentemente este estado representa un puente importante de la corriente migratoria interna hacia la zona fronteriza y la emigración hacia Estados Unidos (Vázquez, 1984). Además, los estudios de flujos migratorios de indocumentados hacia California revelan los mismos estados de origen que la migración interna hacia Baja California (especialmente Jalisco y Sinaloa) (Jones, 1984; Cantú, 1986).

En tanto que existen estudios sobre migración en diferentes épocas históricas y algunos trabajos que han utilizado información en el contexto de origen y destino, hasta la fecha no hay ningún estudio sobre México en el que se hayan descompuesto las variables dependientes de la migración en sus diferentes componentes, tal como la primera mudanza, segunda mudanza y mudanzas subsecuentes. Además, las conclusiones a las que se ha llegado en los estudios anteriores son contradictorias, esto quizá se deba a que la primera mudanza y mudanzas subsecuentes se han agrupado como si se tratara de una sola. Los estudios anteriores asumen que las determinantes de la primera mudanza son las mismas para las subsecuentes. Una hipótesis alternativa es que las determinantes se relacionan de manera diferente con el primer movimiento y con los movimientos subsecuentes.

En este estudio primero se describen las experiencias migratorias de acuerdo con los datos proporcionados por los informantes en la Encuesta Mexicana de Fecundidad (EMF) en México y después se analizan las determinantes de migración utilizando el número total de determinantes. Posteriormente la migración se analiza a través de regresiones logísticas comparando la primera mudanza coh la segunda y subsecuentes mudanzas, la segunda mudanza con las subsecuentes, y la tercera mundanza con las subsecuentes. Este análisis aclara si el agrupamiento de todos los movimientos migratorios en una sola variable dependiente está enmascarando interrelaciones importantes y conduciendo a explicaciones incorrectas sobre las determinantes de migración interna en México y sus implicaciones para otros países en desarrollo.

\section{Fuente de datos}

Esta investigación se basa en los datos de la EMF, que se llevó a cabo en México en julio de 1986 y marzo de 1977 (Secretaría de Programación y Presupuesto, 1978, 1979a,b). Varios estudios sobre migración en México se basan en los datos de la EMF. Estos 
estudios, como muchos otros, generalmente examinan los efectos de migración sobre formación de la familia, edad en la que se contrae matrimonio y fecundidad (Brambila, 1982; Edelfesen y Lee, 1983). Los datos de la encuesta EMF utilizados provienen de una muestra representativa, estratificada y agrupada. El cuestionario de la encuesta consistió de una sección estándar de la EMF, así como de una sección especial sobre la historia de la migración de la esposa y del esposo, el estado de salud de ésta durante la niñez y la maternidad. Se entrevistó un total de 7672 mujeres de las cuales se utilizaron 7310 encuestas para los análisis. Ésta es una de las pocas encuestas representativas de México en la que se incluyen muchas preguntas socioeconómicas y de migración.

El presente estudio compara la migración a nivel individual y las influencias socioeconómicas sobre la migración a nivel individual en las siguientes regiones: a) Baja California, b) el resto de la región noroeste (incluyendo los estados de Baja California Sur, Sinaloa, Sonora y Nayarit), c) Jalisco y d) la región del noreste (incluye Nuevo León y Tamaulipas).

Las regiones se escogieron con base en la presencia de ciudades grandes y que son importantes históricamente como regiones de origen o destino de flujos migratorios. La presencia de ciudades grandes facilita una mejor comparación con los resultados de estudios anteriores realizados por Muñoz et al. y Browning y Feindt, relacionados con la migración hacia las grandes ciudades.

Tres de las regiones escogidas representan, en tamaño, la segunda y tercera ciudades más grandes en 1970: Guadalajara, en Jalisco, y Monterrey, en el noreste, y las ciudades grandes de $\mathrm{Me}$ xicali y Tijuana, en Baja California (Secretaría de Programación y Presupuesto, 1975). Los datos del censo de 1970 son apropiados, ya que es el más cercano a la encuesta EMF. La región noroeste se incluye como una zona con más bajo nivel de urbanización situada entre dos de las otras regiones, Baja California y Jalisco. Su urbanización, basándonos en 2500 agregados urbanos, fue de $0.55 \mathrm{com}$ parada con 0.59 para la nación y 0.72 para las otras tres regiones.

El criterio menos importante para flujos migratorios de importancia histórica es la región de origen o destino. El estudio de Whetten y Burnight (1956) sobre los principales flujos de migración interna de por vida para 1950 , indica la presencia de un flujo mayor de Jalisco y de la región noreste hacia Baja California y muestra que esta región es la receptora para tres flujos migratorios importantes. Jalisco también fue una región importante de origen de emigrantes hacia el Distrito Federal. Estos flujos migratorios continuaron hasta 1950 y 1960 (Butler et al., 1987). Posteriormente, se comparan los resultados para Jalisco y la región no- 
roeste con el fin de determinar si hay similitudes que se puedan atribuir a la clase de regiones receptoras y del mismo modo para el caso de Jalisco y la región noroeste con regiones de emigración.

Además, la edad y el estado civil se controlan, en cada región, de la siguiente manera: casados de 15 a 24, casados de 25 a 34, casados de 35 a 49 y solteros de 15 a 24 . Mujeres solteras de 25 a 34 y de 35 a 49 se excluyeron, ya que el tamaño de $N$ era muy pequeño (menos de 12) para permitir el análisis estadístico multivariado. Ya que la mayoría de las preguntas en este estudio se relacionan con el estado civil, la submuestra de mujeres solteras de 15 a 24 contiene datos para pocas variables, por lo que estas muestras se incluyen únicamente en la descripción y no en el análisis multivariado. Las muestras de agrupamiento por edades representan todos los informantes para la región e incluyen las cuatro submuestras de estado civil, así como también los rangos de edades fuera de las submuestras.

El tamaño de las submuestras se indica en el cuadro 1. Varían en tamaño desde 15 (Baja California, solteros de 15 a 24) hasta 169 (región noreste, casados de 35 a 49). La población total de las cuatro regiones constituyen $21.5 \%$ de la población mexicana en 1970 y $21.1 \%$ en 1980 .

\section{Variables independientes}

Basados en investigaciones anteriores escogimos trece variables independientes para analizarlas. Las definiciones y categorías de las variables dependientes e independientes se encuentran en el cuadro 2 .

Como en muchos de los estudios sobre migración, algunas de las variables independientes están incluidas, aun cuando los eventos migratorios ocurrieron en el pasado (por ejemplo, véase Greenwood, Ladman, y Siegel, 1981; Brown y Goetz, 1987). Esto se justifica, ya que la mayoría de los movimientos en los últimos 10 años fueron relativamente recientes y las características tienden a ser correlaciones positivas con las características de movimientos temporales. Sería preferible que todas las variables independientes ocurrieran antes de los movimientos relevantes, pero este tipo de datos no estaban disponibles.

Los estudios de Muñoz et al. $(1971,1972,1973)$ sobre la ciudad de México muestran que educación, alfabetización, ocupación y tamaño de la residencia actual influian en la migración. El tamaño de la residencia siempre se mide después de la migración. No es el objetivo de este artículo explicar la causa; sin embargo, 


\section{CUADRO 1}

Tamaño de la submuestra tomada de World Fertility Survey, 1967-1977

\begin{tabular}{|c|c|c|c|c|c|}
\hline $\begin{array}{l}\text { Categorías por } \\
\text { edad (años) }\end{array}$ & México & $\begin{array}{c}\text { Estado de } \\
\text { Baja California }\end{array}$ & $\begin{array}{c}\text { Región } \\
\text { noroeste }\end{array}$ & $\begin{array}{c}\text { Región } \\
\text { noreste }\end{array}$ & $\begin{array}{l}\text { Edo. de } \\
\text { Jalisco }\end{array}$ \\
\hline \multicolumn{6}{|c|}{ Informantes casados } \\
\hline $15-24$ & 1468 & 23 & 109 & 89 & 77 \\
\hline $25-34$ & 2143 & 44 & 127 & 142 & 120 \\
\hline \multirow[t]{2}{*}{$35-49$} & 1510 & 90 & 137 & 169 & 124 \\
\hline & 5121 & & & & \\
\hline \multicolumn{6}{|c|}{ Informantes solteros } \\
\hline $15-24$ & 601 & 15 & 39 & 49 & 49 \\
\hline Total ${ }^{*}$ & 7310 & 209 & 470 & 535 & 439 \\
\hline
\end{tabular}

*: Incluye todos los informantes.

es un indicador de propensión de movimiento en el futuro ya que los migrantes frecuentemente planean sus movimientos con anterioridad.

Educación, ocupación y zona de origen (lo cual indirectamente se relaciona con el tamaño de la residencia durante la niñez) fueron importantes en los estudios de Monterrey hechos por Browning/ Feindt $(1968,1969,1979)$. La alfabetización fue significativa en la migración hacia Baja California en los sesenta (Butler et al., 1987).

Para poder determinar los posibles efectos de la fecundidad sobre la migración se incluyeron dos variables acumulativas de fecundidad: los hijos nacidos vivos y los hijos nacidos vivos en los últimos cinco años. Con el fin de controlar el efecto de la variable edad, que se muestra en el cuadro 2, se incluyó en el análisis para todas las muestras (por ejemplo, todas las edades combinadas) para cada región.

Los valores medios de las variables independientes se muestran en el cuadro 3. Se puede observar que por región, la fecundidad tiene menos variación que la migración. Comparando la fecundidad media por región, Jalisco muestra los valores más altos, mientras la región noreste presenta un valor mucho más bajo que la media. Hay un gran incremento en la fecundidad total, aproximadamente un nivel de siete hijos por mujer para la categoría con una edad entre 35 a 49 años. En cuanto a los hijos nacidos en los últimos cinco años, hay valores considerablemente más altos en 
MIGRACIÓN EN MÉXICO

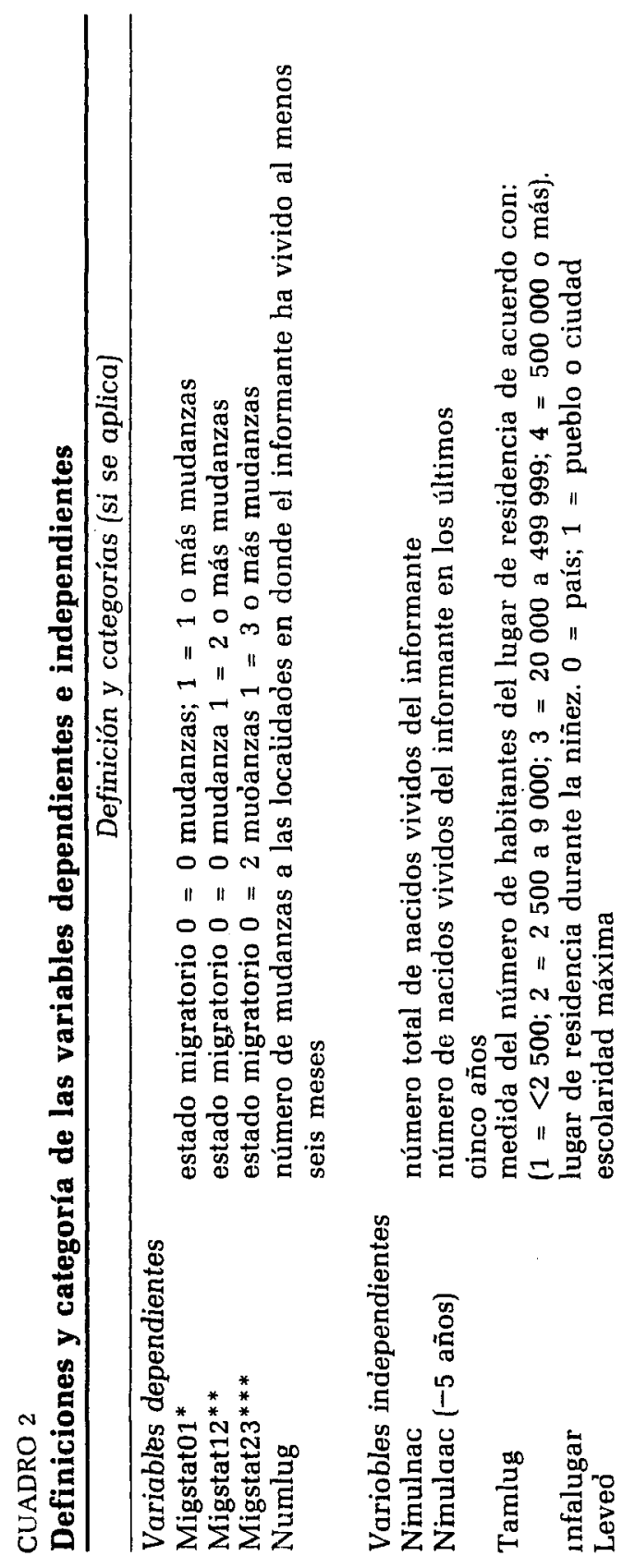




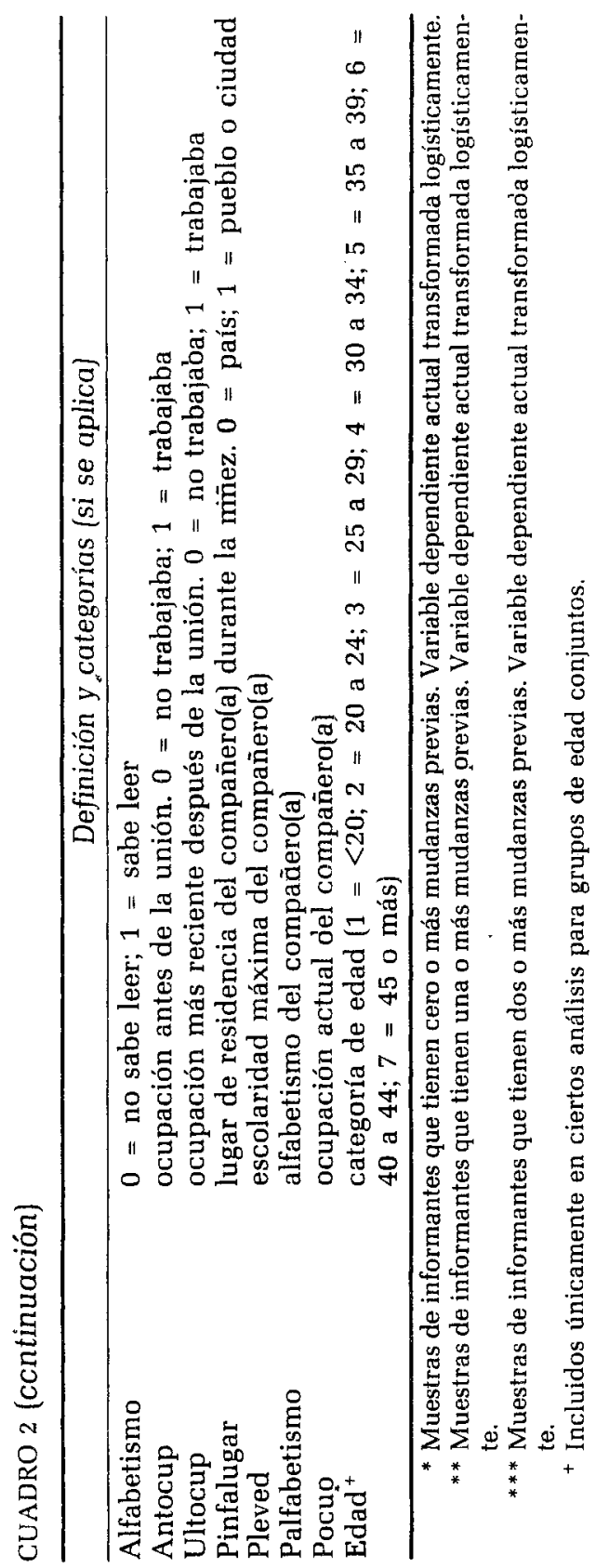


las categorías de 15 a 24 y 25 a 34 disminuyendo en $50 \%$ para el grupo de edad entre 35 a 49 años.

El tamaño de la residencia actual se relaciona con el nivel de urbanización de la respectiva región. Por ejemplo, en la región noroeste, que es una de las regiones menos urbanizadas, tiene la media más baja de tamaño de residencia. Infalugar y Pinfalugar muestran diferencias consistentes entre regiones. Si comparamos por regiones Baja California y Jalisco tienen generalmente los porcentajes más altos para personas de origen de pueblo/ciudad para los dos grupos más viejos. Estos resultados corroboran los resultados de un estudio anterior en el que se muestra que para el periodo 1900 a 1980 la mayoría de los inmigrantes a Baja California provinieron de los lugares urbanizados de Jalisco (Butler et al., 1987). Es muy probable que estos resultados reflejen la migración que ha habido del Distrito Federal hacia Jalisco.

La educación es significativamente más baja en las personas casadas que en las solteras. Esto quizás refleja la posposición o abstinencia del matrimonio en las personas más educadas. Para los casados entre las edades de 15 a 24, los valores se encuentran entre 4.34 en la región noreste a 6.26 en Baja California. Por otra parte, los valores para los solteros de la misma categoría de edad van de 7.71 en Jalisco a 9.27 en Baja California.

En la medida de alfabetización Alfabetismo y Palfabetismo entre regiones Baja California presenta generalmente los valores más altos. Al igual que en educación, varía significativamente de acuerdo con la edad, indicando un menor grado de educación para las cohortes más viejas. Este resultado es consistente con los resultados obtenidos en otras investigaciones (Browning y Feindt, 1969; Muñoz et al., 1973). La alfabetización se aproxima a $100 \%$ en las mujeres solteras, esto refleja en parte, las diferencias de matrimonios por nivel educacional.

Como se muestra en el cuadro 3, entre 51 a $66 \%$ de los informantes trabajaron antes de casarse (variable Antocup), mientras que únicamente 11 a $39 \%$ empezaron a trabajar después de casarse (Ultocup). Quizás se puede inferir que la transición del estado de soltero a casado ha traído como resultado que entre 30 a $40 \%$ de las mujeres no trabajen fuera de casa.

Los resultados para las variables Antocup y Ultocup, cuando se disgregan en detalle en cuanto a ocupación, muestran diferencias sustanciales entre las diferentes regiones. Por ejemplo, para el grupo de edad 25 a 34, los porcentajes de informantes con empleo en la categoria profesional/ejecutivo para las diferentes regiones son 2.3 (Baja California), 4.7 (región noroeste), 5.8 (Jalisco) y 5.6 (región noreste). Para empleados de oficina, los porcentajes 


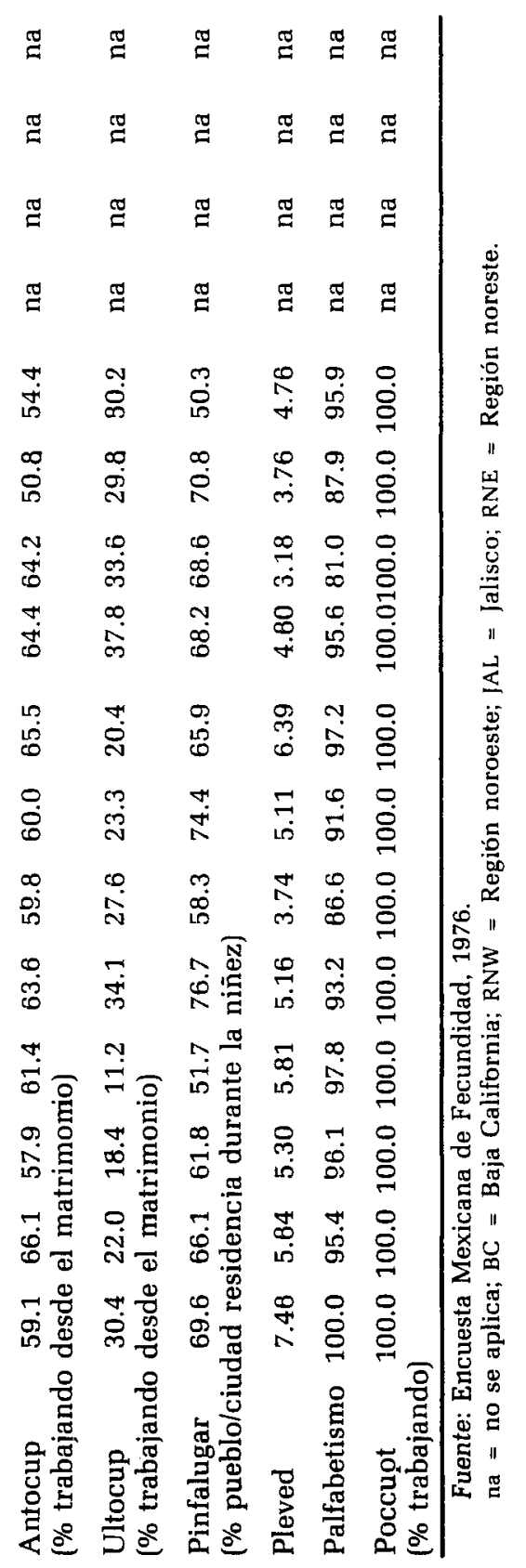


son $9.1,5.5,12.5$ y 18.3, respectivamente. En este trabajo no analizamos estas distinciones más a fondo pero subrayamos lo importante de ellas para futuras investigaciones.

En contraste con la mayoría de mujeres que no trabajaban en ese momento, virtualmente todos los informantes hombres tenían empleo. Los resultados para parejas en que los dos trabajaban son muy altos considerando la alta tasa de desempleo en México (Pick et al., 1987).

Los valores tanto de las variables dependientes como los de las independientes son comparables con los obtenidos en otros estudios del mismo periodo y del censo mexicano de 1970. La variación por región y edad es aparente para algunas variables, por ejemplo educación, alfabetización pero no para otras.

Con el propósito de explicar más a fondo los efectos socioeconómicos sobre la migración, se realizaron dos análisis multivariados: a) Un análisis de regresión utilizando como variable dependiente la frecuencia de migración (por ejemplo, número de mudanzas) y b) un análisis de regresión logística de las tres variables dependientes del estado migratorio.

\section{Experiencias migratorias}

Cuatro variables dependientes se examinan en este análisis. La variable Numlug es el número de lugares en los que el informante ha vivido durante su vida. La variable Migstato1 representa una relación desigual, donde $P$ es la probabilidad, para cada persona que no se ha mudado, de hacer 1 o más mudanzas con una duración mínima de 6 meses; por lo que puede tener dos posibles valores, 0 = ninguna mudanza (por ejemplo, no migrante) y $1=10$ más mudanzas (por ejemplo, migrante). El cambio de lugar puede ocurrir durante cualquier etapa de la vida. Aunque estudios anteriores en muchos países han mostrado consistentemente que las tasas de migración más altas ocurren entre 20 y 30 años (Bogue, 1985 entre otros). Ya que los dos grupos más jóvenes de informantes se sobrelapan con edades altamente migratorias, las variables dependientes se enfocan en mudanzas recientes.

La tercera variable dependiente, Migstat12, es una relación desigual, donde $P$ representa la probabilidad de que una persona que se haya mudado una vez tenga dos o más mudanzas con una duración mínima de 6 meses. Sus dos posibles valores son: $0=$ una mudanza y 1 = dos o más mudanzas. Por lo tanto, Migstat12 es por definición un subconjunto condicional de la muestra original. La variable dependiente Migstat12 se selecciona para deter- 
minar cuáles son las influencias socioeconómicas que afectan el movimiento después que se ha hecho el primer movimiento.

Una tercera variable dependiente, Migstat23, es una relación desigual donde $P$ es la probabilidad de que una persona que se ha mudado dos veces se mude una tercera o más veces con una duración mínima de 6 meses. Una vez más, Migstat23 es por definición un subconjunto condicional de la muestra para Migstat12. Esta variable es utilizada para determinar que lo que afecta a las órdenes más altas de movilidad, una vez que la segunda mudanza se haya hecho. Las reducciones en el tamaño de muestra condicional para Migstat23 son tales que únicamente se pueden realizar los análisis de regresiones logísticas si se unen los grupos de diferentes edades por región. Los análisis de las variables subsecuentes Migstat45, etc., no se pueden realizar debido a que hay una mayor reducción en el tamaño de muestra. Por lo que únicamente nos referiremos a Migstat1, Migstat12 y Migstat23 a pesar de que las variables en las subsecuentes regresiones logísticas son las transformaciones logísticas de las relaciones desiguales de las tres variables.

La relación de todos los movimientos para las regiones es la siguiente:

Porcentaje de distribución del número de mudanzas EMF 1976 a 1977

\begin{tabular}{lrrrrrrrrc}
\hline Núm. de mudanzas & 0 & $\mathbf{1}$ & 2 & 3 & 4 & 5 & $6+$ & Tot & $\begin{array}{c}\text { Núm. medio } \\
\text { mudanzas }\end{array}$ \\
\hline Baja California & 7 & 34 & 30 & 15 & 6 & 3 & 5 & 100 & 2.19 \\
Región noroeste & 23 & 28 & 24 & 12 & 6 & 3 & 4 & 100 & 1.77 \\
Jalisco & 26 & 34 & 20 & 10 & 6 & 2 & 2 & 100 & 1.55 \\
Región noreste & 27 & 32 & 22 & 11 & 5 & 1 & 2 & 100 & 1.56 \\
Total & 24 & 32 & 23 & 11 & 5 & 2 & 3 & 100 & 1.77 \\
\hline
\end{tabular}

Si analizamos la muestra total observamos que una cuarta parte de los informantes no se mudó, mientras que $55 \%$ experimentó una o dos mudanzas. Para más de dos mudanzas, existe un patrón decreciente exponencial. Los informantes de Baja California fueron los que más migraron, con muy pocos no migrantes y el orden más alto $(3+)$ de migrantes en cualquier región.

Existen diferencias regionales que son aparentes para el número de mudanzas, por edad: 
Número de lugares en los que vivieron los informantes por lo menos seis meses

\begin{tabular}{lccccc}
\hline Edad & $\begin{array}{c}\text { Baja } \\
\text { California }\end{array}$ & $\begin{array}{c}\text { Begión } \\
\text { noroeste }\end{array}$ & Jalisco & $\begin{array}{c}\text { Región } \\
\text { noreste }\end{array}$ & Media \\
\hline 15 a 24 & 1.70 & 1.60 & 1.24 & 1.34 & 1.47 \\
25 a 34 & 2.16 & 1.83 & 1.72 & 1.69 & 1.85 \\
35 a 49 & 2.71 & 1.89 & 1.70 & 1.64 & 1.98 \\
Media & 2.19 & 1.77 & 1.55 & 1.56 & 1.77 \\
\hline
\end{tabular}

Consistentemente Baja California ha tenido el número más alto de mudanzas. La razón quizás se debe a que Baja California se utiliza como un puente hacia Estados Unidos. Este resultado es consistente con el estudio hecho por Winnie (1981), en el cual se indica que durante los sesenta, Baja California tenía tasas de crecimiento relativamente más altas que Jalisco y la región noreste y tasas casi iguales a las de la región noroeste. Se podría esperar que el número total de mudanzas incrementara con la edad, como en el caso de Baja California, en donde hay la media mudanza de incremento en cada categoría de edad. Sin embargo, para las otras tres regiones, hay únicamente un ligero incremento en el número de mudanzas. Esto muestra que los flujos migratorios históricos hacia Baja California han existido desde hace tiempo.

La variable dependiente Migstat01 está representada en porcentaje de migrantes. El porcentaje de no migrantes para las diferentes regiones se muestra en la siguiente tabla:

Porcentaje de no migrante, EMF, 1976 a 1977

\begin{tabular}{lccccc}
\hline Edad & $\begin{array}{c}\text { Baja } \\
\text { California }\end{array}$ & $\begin{array}{c}\text { Begión } \\
\text { noroeste }\end{array}$ & Jalisco & $\begin{array}{c}\text { Región } \\
\text { noreste }\end{array}$ & Media \\
\hline 15 a 24 & 17.4 & 22.9 & 32.9 & 28.1 & 25.3 \\
25 a 34 & 4.5 & 19.7 & 20.0 & 22.5 & 16.7 \\
35 a 49 & 2.2 & 20.9 & 18.5 & 13.6 & 14.0 \\
Media & 8.0 & 21.5 & 23.8 & 21.4 & 18.7 \\
\hline
\end{tabular}

Existe una variación importante por edad y región donde Baja California tiene una migración mucho mayor que cualquiera de las otras regiones, reflejando el rápido crecimiento y la alta tasa de inmigración para Baja California, 1930 a 1970 . Esto es especialmente evidente para los grupos de edad más avanzada, con más de $\mathbf{9 5 \%}$ de migrantes. Baja California, Jalisco y la región noreste 
muestran decrementos significativos por edad en porcentaje de no migrantes, mientras que en la región noroeste no hay un cambio perceptible en el porcentaje de no migrantes.

Debido a la naturaleza retrospectiva de los datos, cambios por los grupos de edad reflejan cambios en cohortes. Para las cuatro regiones se pueden anticipar decrementos en el porcentaje por edad de no migrantes ya que, presumiblemente, son equivalentes a los incrementos relacionados con cohorte.

La variable dependiente Migstat12 se puede resumir con los valores porcentuales de la tasa de informantes que realizaron $2+$ mudanzas comparado con aquellos que hicieron $1+$ mudanzas.

Relación porcentual de mudanzas $2+$ a $1+$

\begin{tabular}{lccccc}
\hline Edad & $\begin{array}{c}\text { Baja } \\
\text { California }\end{array}$ & $\begin{array}{c}\text { Región } \\
\text { noroeste }\end{array}$ & Jalisco & $\begin{array}{c}\text { Región } \\
\text { noreste }\end{array}$ & Media \\
\hline 15 a 24 & 68.4 & 66.7 & 44.9 & 50.0 & 57.5 \\
25 a 34 & 69.0 & 64.2 & 61.1 & 53.8 & 62.0 \\
35 a 49 & 70.4 & 78.1 & 53.1 & 54.4 & 64.0 \\
Media & 69.3 & 69.7 & 53.0 & 52.7 & 61.2 \\
\hline
\end{tabular}

Comparando las regiones, la tasa media de Baja California de $2+$ a $1+$ mudanzas es elevada, como si fuese la tasa de $1+$ a 0 mudanzas. Estos resultados reflejan la tendencia de inmigrantes a Baja California de tener mudanzas repetidas, lo cual quizás refleje paradas intermedias de la migración del centro de México hacia zonas fronterizas incluyendo la emigración hacia Estados Unidos e inmigración de regreso a México. Además, para Baja California existe una prevalencia alta de migrantes que se mueven continuamente (Baja California tiene $36 \%$ más de migrantes $3+$ que las otras tres regiones).

También la región noroeste tiene una tasa alta de migrantes $2+$ a $1+$, aunque no es lo mismo para $1+$ a 0 mudanzas. La tasa media para las cuatro revela un incremento constante por edad en la proporción $2+$ a $1+$. Dos regiones difieren de este patrón: Baja California en la que no hay un cambio de edad perceptible y Jalisco con un patrón variable.

La variable dependiente Migstat23 se puede resumir de la siguiente manera: la relación porcentual del número de informantes que tiene $3+$ a $2+$ mudanzas (resultados que son específicos para grupos de edad no se dan debido al pequeño tamaño de $N$ ). 
Relación porcentual de mudanzas $3+$ a $2+$

\begin{tabular}{ccccc}
\hline $\begin{array}{c}\text { Baja } \\
\text { California }\end{array}$ & $\begin{array}{c}\text { Región } \\
\text { noroeste }\end{array}$ & Ialisco & $\begin{array}{c}\text { Región } \\
\text { noreste }\end{array}$ & Media \\
\hline 54.7 & 50.8 & 52.5 & 46.6 & 51.1 \\
\hline
\end{tabular}

Las diferencias entre regiones son pequeñas, Baja California presenta la relación más alta y la región noroeste la más baja. La tasa media es de $16 \%$ más baja que la media de la relación $2+\mathbf{a} 1+$.

\section{Análisis de regresión}

Se llevó a cabo un análisis de regresión lineal para la variable dependiente Numlug, definida como el número de lugares donde un informante ha vivido por lo menos 6 meses, con valores posibles en un rango de 0 a 6 o más. Esta variable se relaciona con otras variables dependientes, cada una de las cuales representa un aspecto categórico de la variable numérica Numlug. Ésta se incluye con el fin de proveer información general del número de movimientos. Debido al rango limitado de valores numéricos los resultados deben verse cautelosamente (Bieber, 1988).

Las regresiones lineales y las regresiones logísticas se basan en el mismo grupo de variables independientes, explicado anteriormente. La edad se incluye como variable independiente únicamente para muestras donde hay agrupamiento por grupos de edad. El análisis de regresión se basa en un modelo de análisis de regresión lineal simple:

$$
y=a+b_{1} x_{1}+b_{2} x_{2}+b_{n} x_{n}+e
$$

donde

$\mathrm{y}=$ variable dependiente

$\mathrm{x}_{1}, \mathrm{x}_{2}, \ldots \mathrm{x}_{n}$ son las variables independientes

$b_{1}, b_{2}, \ldots b_{n}$ son los coeficientes correspondientes a las variables independientes $x$

$\mathbf{e}=$ error aleatorio

Los objetivos del análisis por regresión son examinar si existen influencias regionales y de edad sobre la frecuencia de migración y comparar los resultados de propensión a migrar entre la regresión simple y la regresión logística. 
La variable dependiente Numlug, es la mejor variable de EMF disponible para medir frecuencia de migración. En la revisión bibliográfica no se encontró ningún otro estudio sobre migración en México en el que se considere frecuencia como una variable dependiente. Algunos investigadores han propuesto que existe un gran conjunto de trabajadores migratorios en México que han experimentado mudanzas repetitivas. Ya que Numlug es una medida de por vida, algunas mudanzas pueden haber ocurrido a edades muy tempranas. Aunque la mayoría de los movimientos se llevan a cabo entre 20 y 30 , por lo que esta variable se enfoca a movimientos recientes.

En el análisis de regresión lineal, el criterio mínimo para considerar variables independientes fue el de un nivel de $F$ de 1.0. El número de pasos seleccionado varía de acuerdo con la submuestra.

Los resultados de la regresión para frecuencia de migración, para las diferentes grupos de edad conjuntos se muestran en el cuadro 4. Para la región noroeste se muestran los grupos de estancia durante la niñez (cuadro 4). Para la región noroeste la residencia durante la niñez es lo más importante. Nuestra interpretación es que refleja la importancia de la migración masiva de zonas rurales a urbanas que se llevó a cabo en las décadas de los años cuarenta, cincuenta y sesenta (véase Unikel, 1977; Scott, 1982). La última ocupación también es importante pero tiene un menor efecto positivo. En la región noroeste, los resultados de la regresión corresponden a dos efectos dominantes: residencia durante la niñez y última ocupación, los cuales se discutirán para la regresión logística de $0-1+$ y 1-2 .

Para Jalisco el predictor más importante y positivo para número de mudanzas es la ocupación anterior. Mientras que en la región noreste, el efecto más importante es el nivel educacional del compañero(a). La correspondencia no es con la altamente significativa Influgar para regresión logística 0-1 +, pero con el resultado similar menos significativo para la regresión logística 1-2 + . Aunque Pinfalugar (correlacionado altamente con Infalugar) es segundo en importancia en la regresión. Ultocup es tercera en significancia en la regresión y corresponde al efecto significativo en la regresión logística 1-2 + . En general, los resultados de la regresión para la región noreste sintetizan los efectos mayores que se discutirán en las regresiones logísticas.

Los efectos de la fecundidad sobre la migración son menores y viceversa, la migración sobre la fecundidad (Pick et ol., 1988). El resultado general de las débiles interacciones entre fecundidad y migración contradice las fuertes interacciones observadas por Brambila (1982) y Ellefsen y Lee (1983), quienes también utiliza- 
ESTUDIOS DEMOGRÁFICOS Y URBANOS

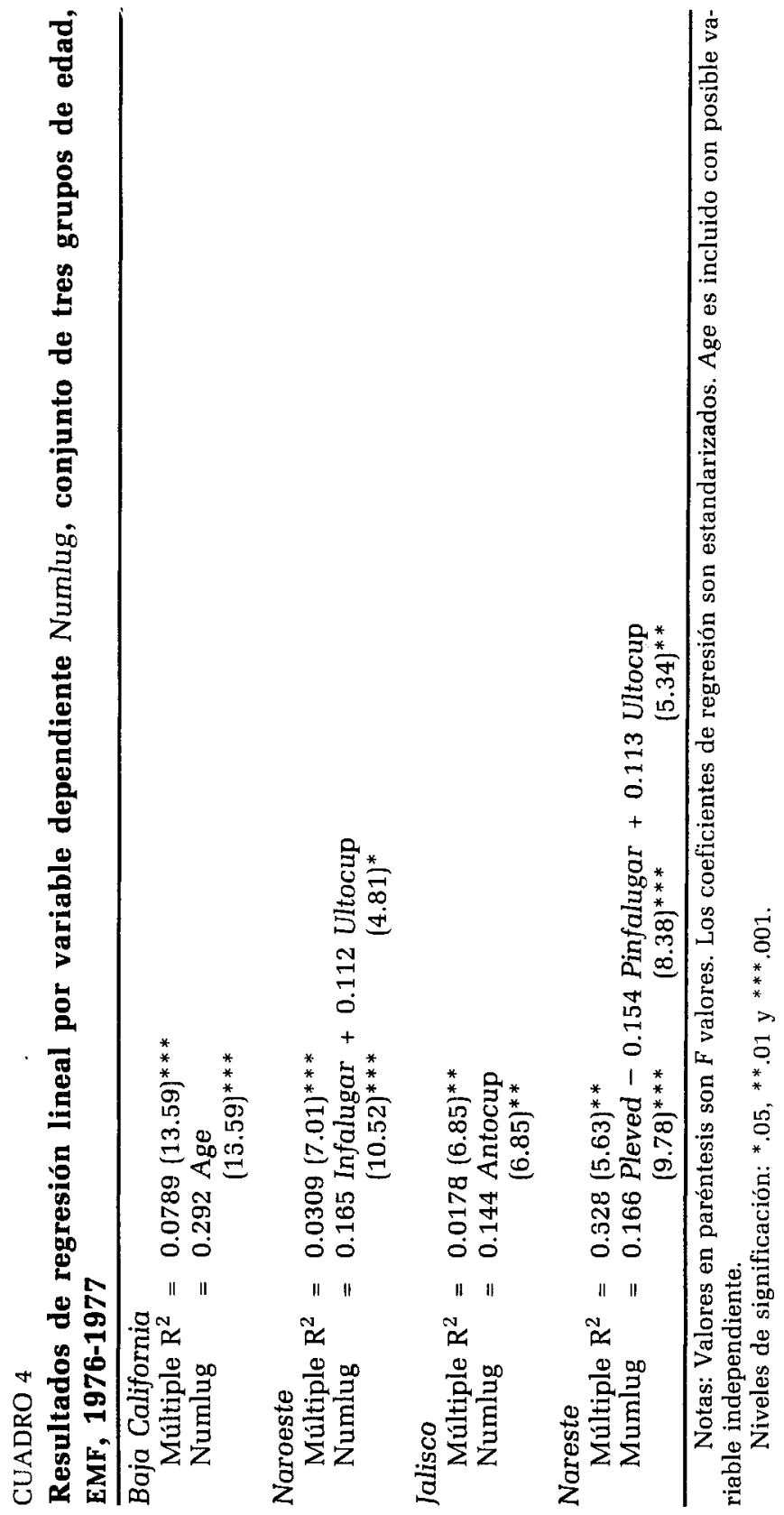


ron los datos de la EMF pero para otras regiones de México. Quizá tales interacciones son características de algunas regiones y flujos migratorios de México pero no para otras.

En general los resultados del análisis de regresión para los grupos de edad conjuntos corresponden en la región noroeste a un efecto muy bien conocido de migración de zonas rurales a urbanas. En las otras regiones hay efectos positivos de la ocupación femenina y efectos educacionales contradictorios. Aunque estos resultados son útiles no distinguen las diferentes categorías de los migrantes. Esto es, no revelan cambios posibles en las influencias socioeconómicas sobre la migración conforme al progreso de categoría no migrante a primero, segundo y más altos órdenes de mudanza. En México y otras naciones es importante examinar si estos efectos cambian o no de acuerdo al progreso de los estados de la movilidad.

\section{Análisis de regresión logística}

El análisis de regresión lineal logística se llevó a cabo para las siguientes variables dependientes: Migstat1, Migstat12, Migstat23 y el grupo completo de variables independientes Edad para el conjunto agregado de diferentes grupos de edad. El valor mínimo de significancia para las variables independientes fue de $F=0.10$. El número de pasos seleccionados para la regresión logística varió entre uno y cinco, con un orden de entrada de las variables independientes determinado por un nivel de significancia de chicuadrada mejorado. El valor mejorado de chi-cuadrada se define como el log de la relación del valor actual con la función probabilística previa (Dixon et al., 1985). El conjunto total de chicuadrada mejorado se utiliza para indicar la significancia general de la regresión logística. La prueba de bondad de ajuste es inapropiada, ya que, algunas de las variables independientes son numéricas. Los puntos de parada se determinaron por el balance de un modelo sencillo y la importancia de las variables independientes estimada por los investigadores.

La ventaja del análisis de regresión es que se pueden determinar las variables que predicen mejor el número de mudanzas. Aunque, el análisis de regresión múltiple es frecuentemente considerado inapropiado para variables dependientes categóricas que presentan una fuerte concentración de valores en relativamente pocas categorías (Bieber, 1988). En el presente análisis, las categorías de la variable dependiente representan probabilidades de movilidad, de tal forma que la regresión logística es una técni- 
ca adecuada. La regresión logística se ha utilizado previamente para el análisis de una variable dependiente de migración con dos categorías: no migrante y migrante (Brown y Goetz; 1987). La naturaleza de los datos de nuestro estudio permite un análisis más profundo ya que las variables dependientes se distribuyen en movimientos de transición particulares: 0 a $1+, 1$ a $2+$ o 2 a $3+$.

El modelo estándar de regresión logística es el siguiente:

$$
\log (P / 1-P))=a+b_{1} x_{1}+b_{2} x_{2}+\ldots b_{n} x_{n}+e
$$

donde

$P=$ probabilidad de que ocurra un evento

$\mathbf{a}=$ constante

$\mathbf{e}=$ error aleatorio

$\mathrm{X}_{1}, \mathrm{x}_{2}, \ldots \mathrm{x}_{n}$ son variables independientes

$b_{1}, b_{2}, \ldots b_{n}$ son los coeficientes correspondientes a las variables independientes

El objetivo principal del análisis de la regresión logística es determinar hasta qué punto y qué tipo de región y edad afecta la propensión a migrar. La regresión logística revela efectos socioeconómicos sobre la probabilidad de mudarse para los informantes con cero mudanza, uno y dos mudanzas por región actual de residencia. Para los estados de movilidad de cero y uno, los resultados se dividen en más categorías por edad.

El análisis de regresión logístico para las tres categorías por edad juntas se presenta en los cuadros 5 a 7 . El resultado de Migstat01 difiere entre Baja California y las otras tres regiones (Tabla 5). Para Baja California, el efecto positivo más importante es la edad, aunque la educación ejerce un efecto negativo con una significancia muy similar. Nuestra interpretación es que posteriormente el efecto también refleja edad. La razón es el avance sustancial en los niveles de educación nacional desde 1950 (Muñoz Izquierdo, 1973). Por lo que no es sorprendente que el nivel educacional del informante se correlacione altamente con la edad ( $\mathrm{r}=$ $0.158, p=0.05$ ). Para otras regiones domina un efecto negativo para Infalugar y una relación positiva con Tamlug.

El efecto negativo de Infalugar quizás refleja los grandes flujos migratorios de zonas rurales a zonas urbanas. Desde 1940 hasta 1970, la proporción de la población urbana de México incrementó de 20 a $45 \%$, mientras que la población rural decreció de 72 a $47 \%$, con un componente mixto remanente fijo de $8 \%$ (Unikel, 1977). Por lo tanto, la residencia rural durante la niñez incrementa la propensión a migrar. La relación positiva de Tamlug tam- 
MIGRACIÓN EN MÉXICO

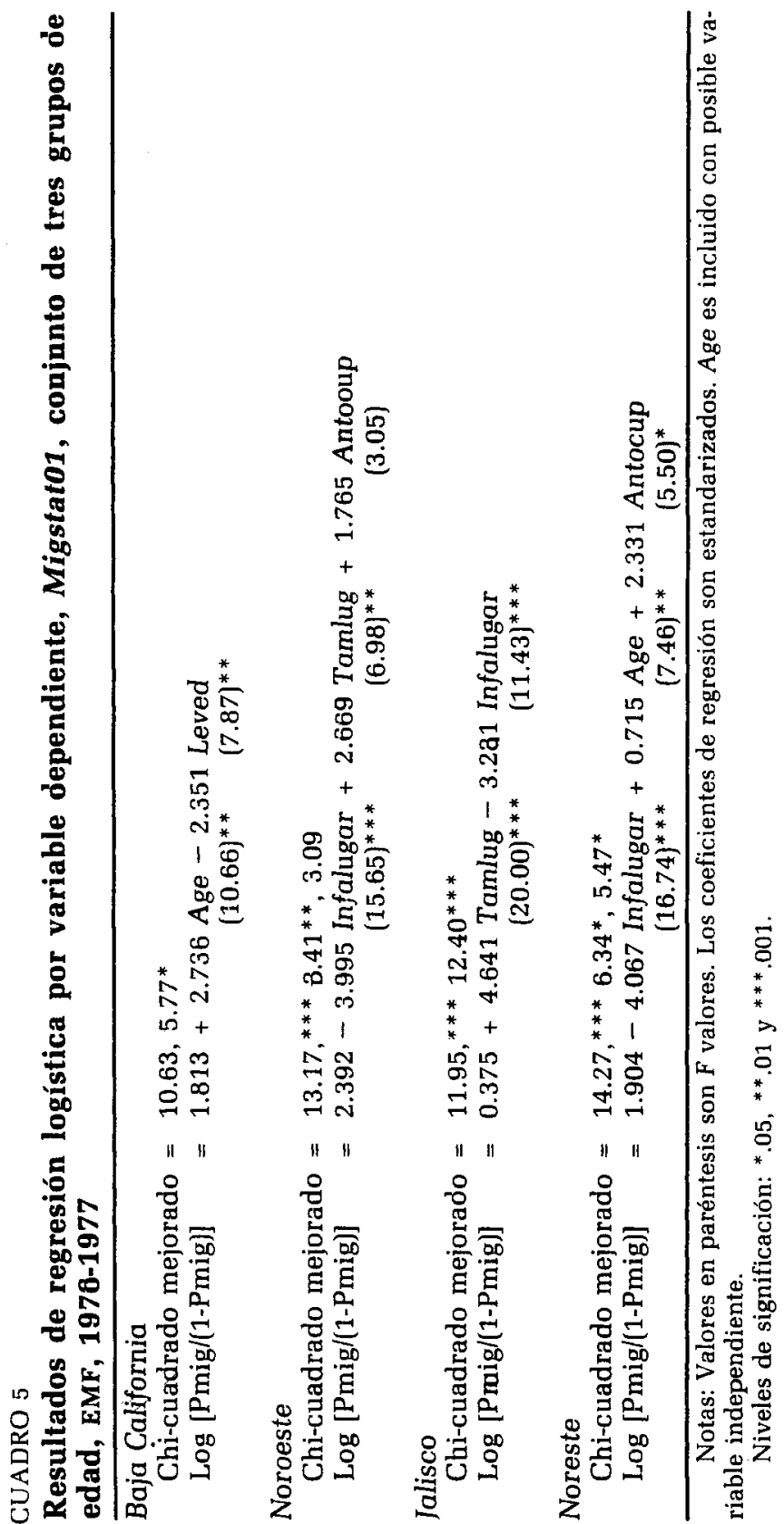




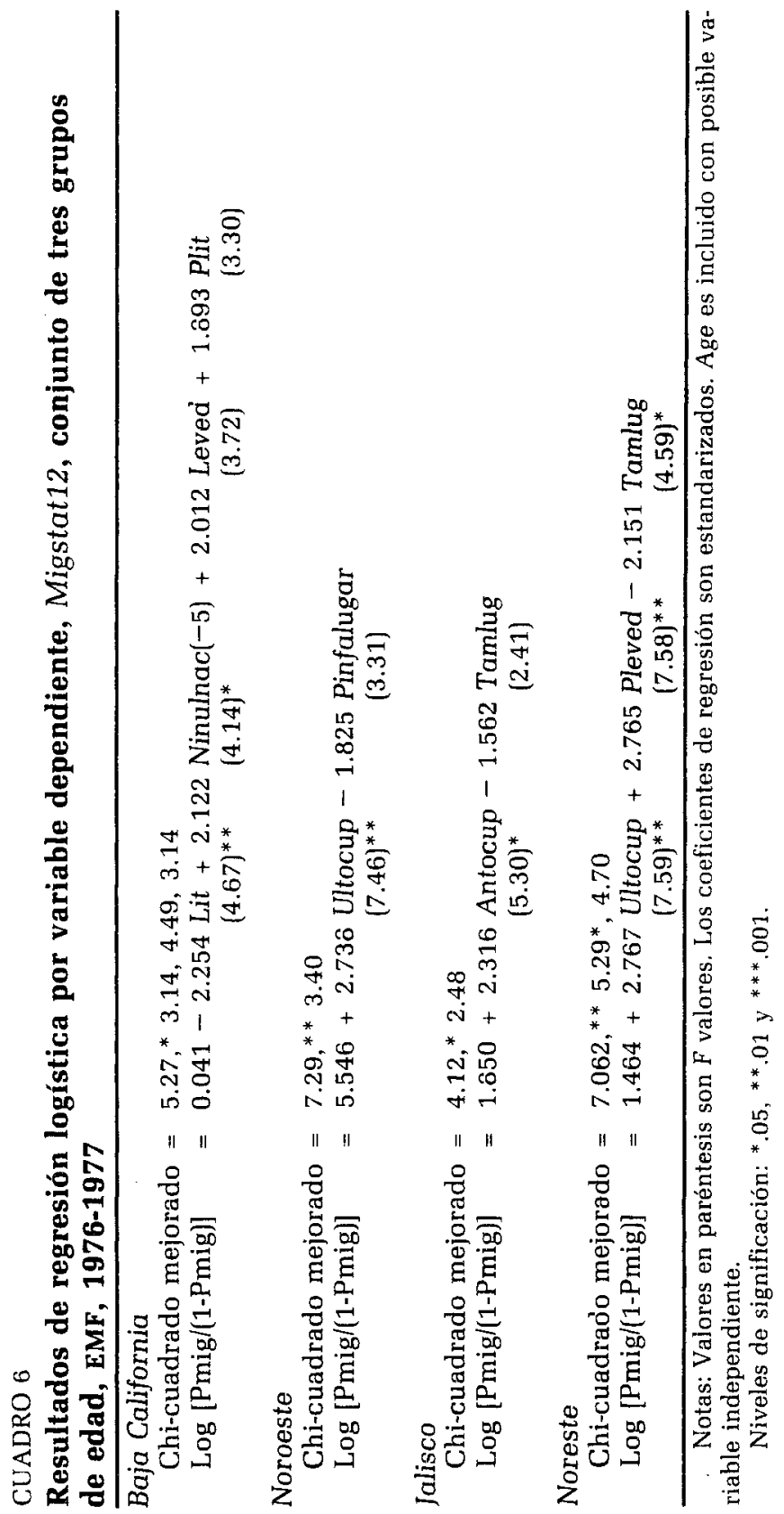


MIGRACIÓN EN MÉXICO

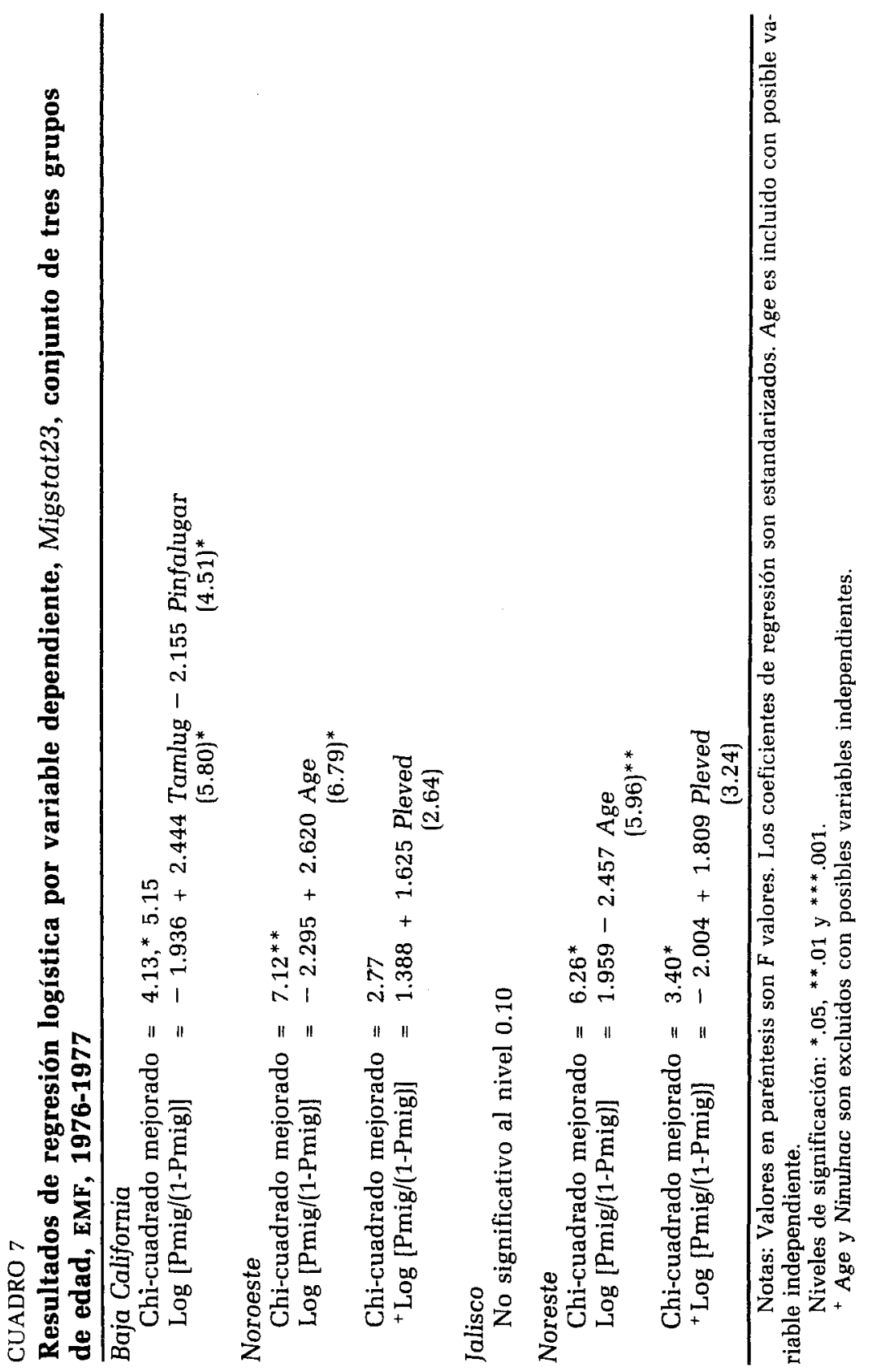


bién refleja esta tendencia. Ocurriendo después de la migración, la residencia actual es indicador de disponibilidad a migrar anteriormente. Ambas reflejan anticipación del lugar de residencia posterior y una probable correlación con el o los lugares de residencia anteriores a su(s) mundanza(s). En general nuestros resultados confirman la importancia del origen rural como una influencia sobre la migración permanente. La carencia de tales efectos significativos para Migstat01 en Baja California quizás refleja el mayor flujo migratorio proveniente de Jalisco, región altamente urbanizada, a Baja California.

Los resultados para Migstat12 enfatizan el factor ocupación, son seguidos por el lugar de residencia y educación. Baja California es una excepción a este patrón, ya que el efecto más importante es negativo y es el de alfabetización. Consideramos que el efecto de alfabetización así como el de educación para la transición 0 $1+$, refleja edad. El efecto positivo de la maternidad en los últimos cinco años es inexplicable y quizás sea un efecto falso. El efecto positivo no significante del nivel de educación es contrario al argumento anterior de alfabetización. El efecto positivo de alfabetización del compañero(a) (también presente para educación en la región noreste) refleja un incremento de la importancia del alfabetismo del compañero(a) para volver a mudarse después de la primera vez. Las explicaciones posibles incluyen que el tener un compañero alfabeto incrementa la habilidad de colectar información acerca de posibles destinos de migración y mayor posibilidad de encontrar trabajo en el lugar de destino.

Para las otras tres regiones, la presencia de una ocupación en el lugar de destino para el informante es la influencia más significante para mudarse después de la primera vez. Además, la posibilidad de que las mujeres casadas puedan trabajar quizás motiven migraciones para que ellas también trabajen, generalmente medio tiempo, en el lugar de destino. Este efecto es más importante para la segunda mudanza que para la primera o mudanzas posteriores a la segunda. Otros resultados significativos para Migstat12 se nota en la región noreste: un efecto positivo del nivel de educación del compañero(a) y una interacción negativa del tamaño del lugar de residencia.

Para Migstat23 en Baja California, la interacción positiva del tamaño del lugar de residencia y el efecto negativo del lugar de residencia durante la niñez asemeja mucho los resultados de Migstat01 para otras regiones. Por lo tanto, para Baja California el flujo migratorio de zonas rurales a urbanas es más importante para los movimientos que se hacen después de las dos primeras mudanzas. En Baja California, el efecto usualmente dominante ru- 
ral a urbano es eclipsado por edad educación/alfabetización y fecundidad para $0-1+$ y 1-2 + mudanzas, pero reaparece después del segundo movimiento.

Para la región noreste, un efecto positivo de edad es el dominante. Para la región noroeste, la edad es más importante pero en dirección negativa. Para probar que otros efectos pueden estar presentes, estas dos regresiones logísticas se volvieron a correr pero eliminando edad y número total de hijos, el cual se correlaciona altamente con edad. Los resultados para la región noroeste mostraron un efecto positivo pero no significante para el nivel de educación del compañero(a), mientras que los resultados para la región noreste, para el mismo factor, muestran un efecto negativo pero también no significante.

\section{Efectos específicos de la edad}

Los resultados de las regresiones logísticas, para tres grupos de edad se discuten en esta sección. Se enfatizan las diferencias de los resultados del agrupamiento de las categorías de edad anteriormente discutidos. Los resultados específicos para la edad se discutirán para los movimientos transitorios de 0-1 + y 1-2 + , pero no para 2-3 + , ya que, el tamaño de muestra es muy pequeño y no permite el análisis multivariado. Los resultados se presentan en los cuadros 8 y 9.

\section{Efectos específicos de la edad para movimientos transitorios 0-1}

Las dos variables independientes más importantes para el conjunto de grupos de edad son el efecto negativo de Inbalugar y la interacción positiva de Tomlug. Estos impactos son muy fuertes en los grupos de diferentes edades particulares de las diferentes regiones. En la región noroeste son fuertes para el grupo de edad 35 a 49; en Jalisco para los grupos de edades de 15 a 24 y 35 a 49 y en el noreste para el grupo de edad de 25 a 34 . La especificidad para cada región quizás refleje la influencia histórica particular en grupos de ciertas edades de migración rural a urbana. La validación de esta hipótesis requiere de datos detallados sobre los flujos migratorios regionales rural a urbano para el periodo 1927 a 1961. Sin embargo, es muy probable que estos datos no existan. Hay algunos otros efectos significativos relacionados con la edad, aunque sin ninguna explicación. Los efectos negativos del nivel educacional del compañero(a) para las regiones noroeste y 


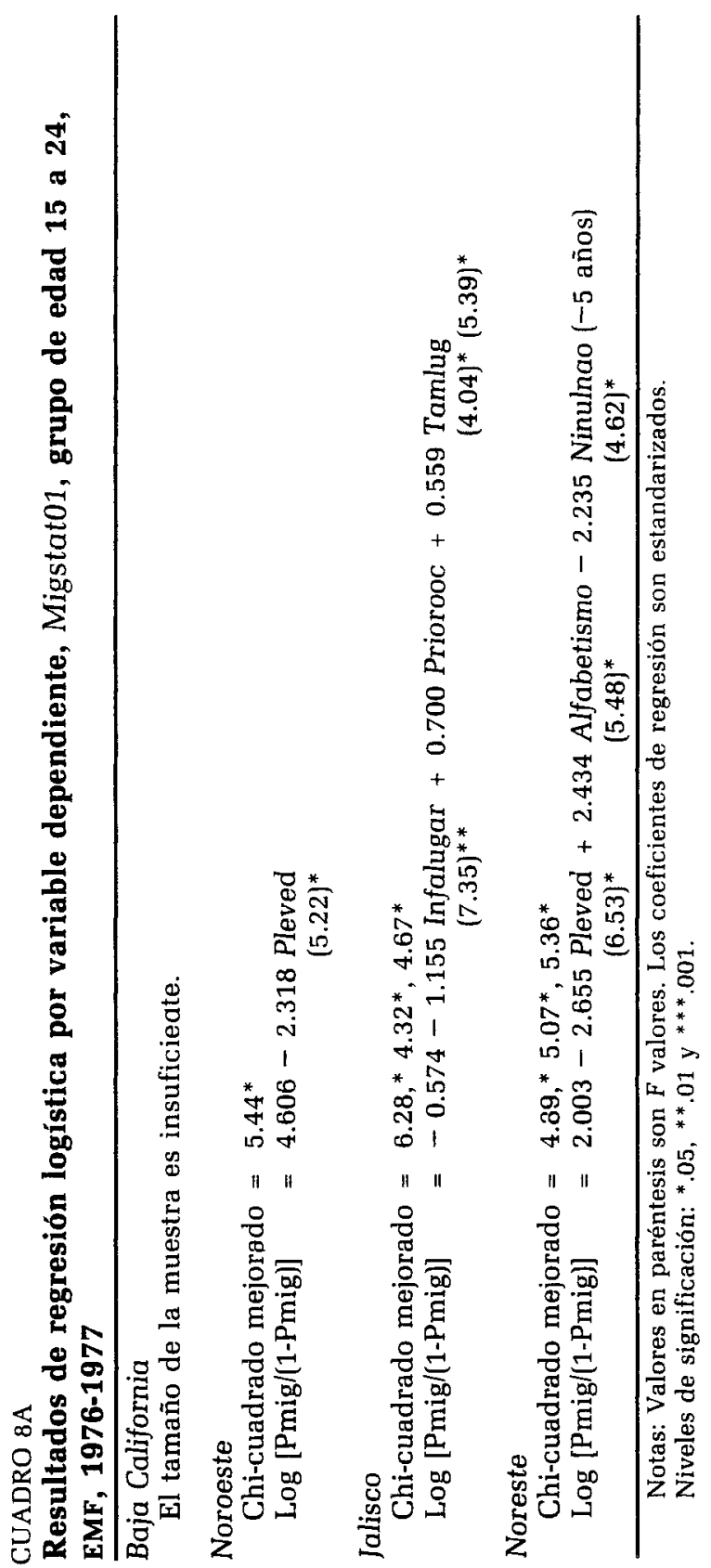




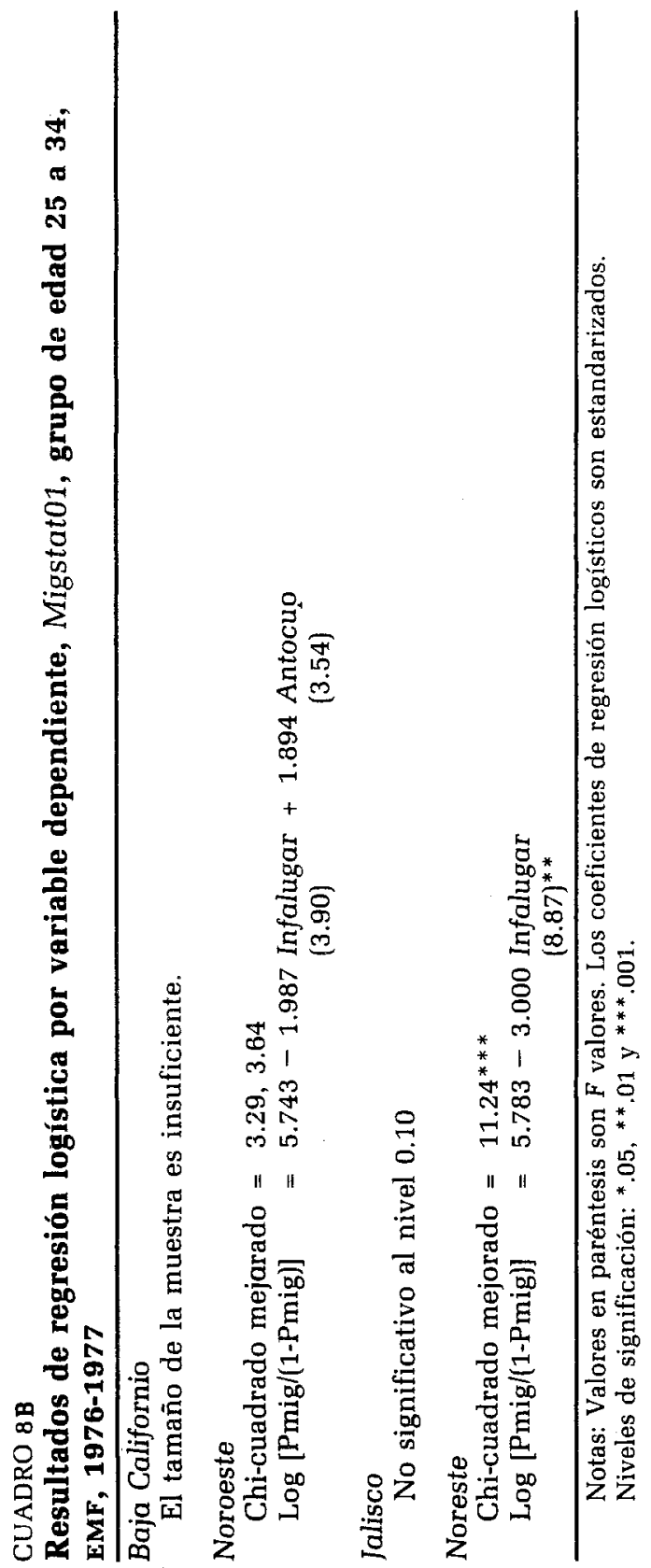




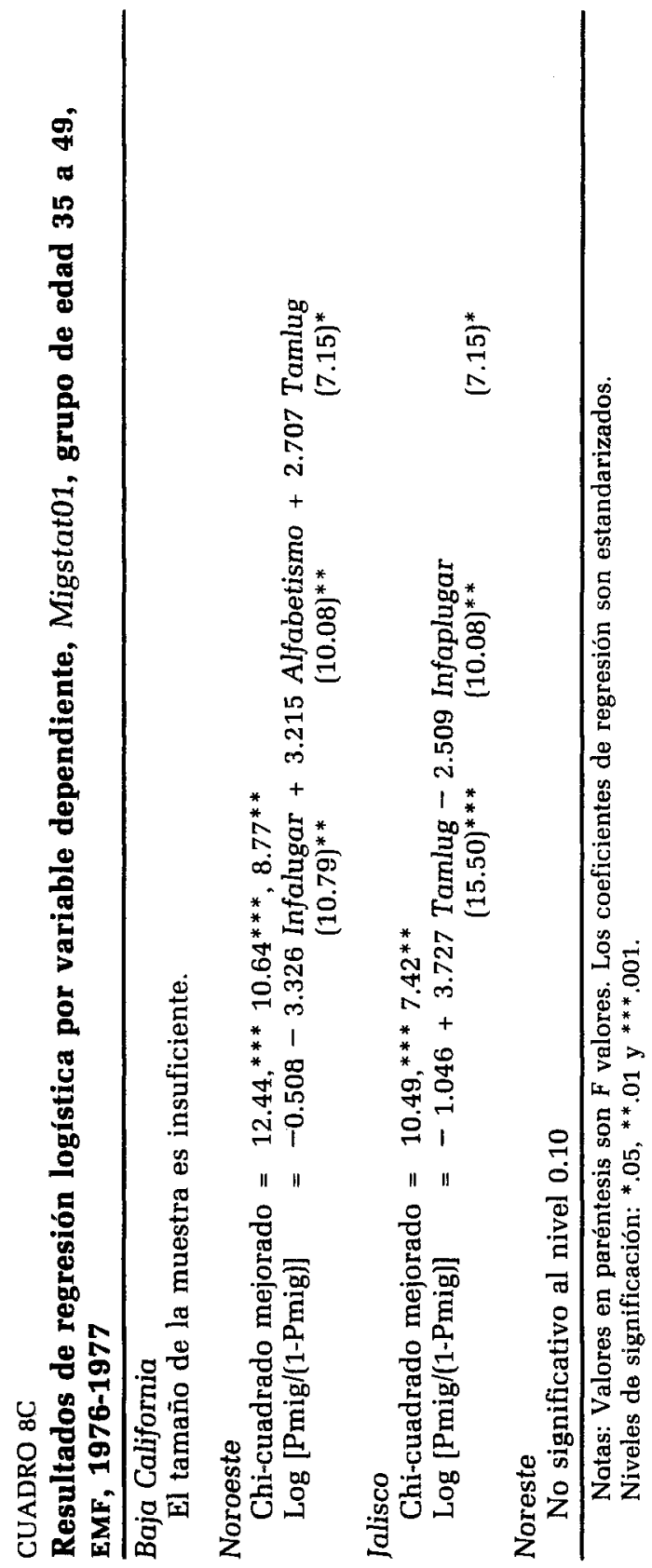




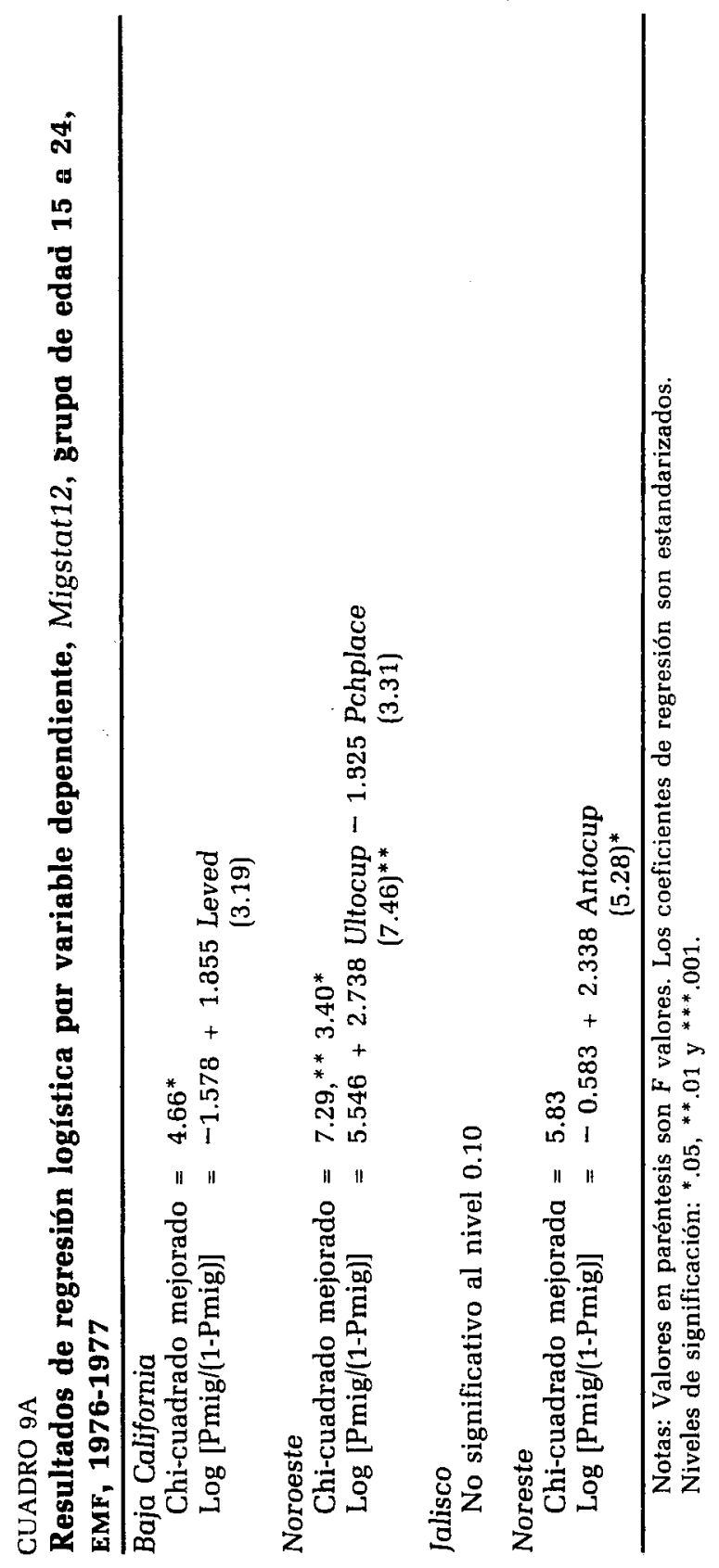




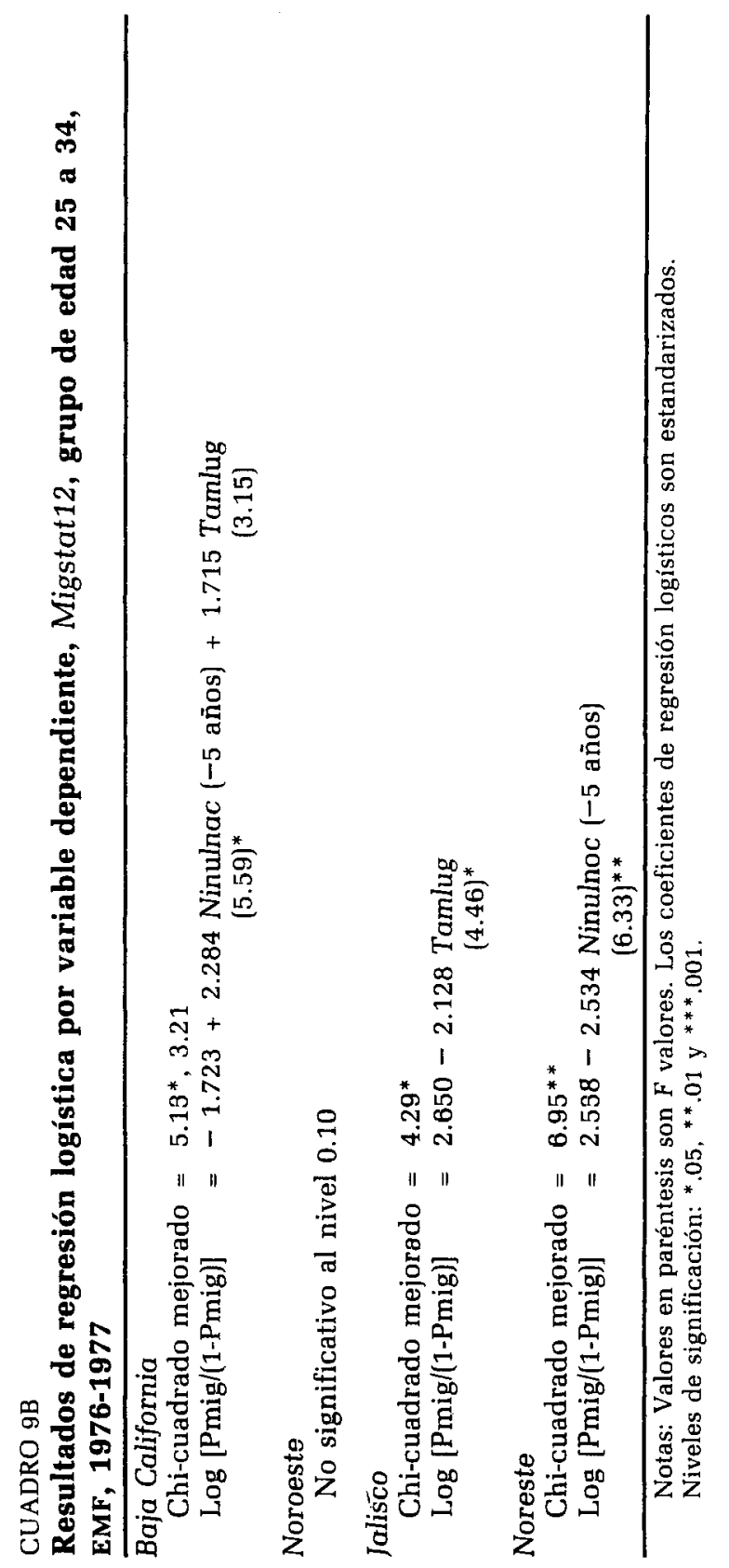


MIGRACIÓN EN MÉXICO

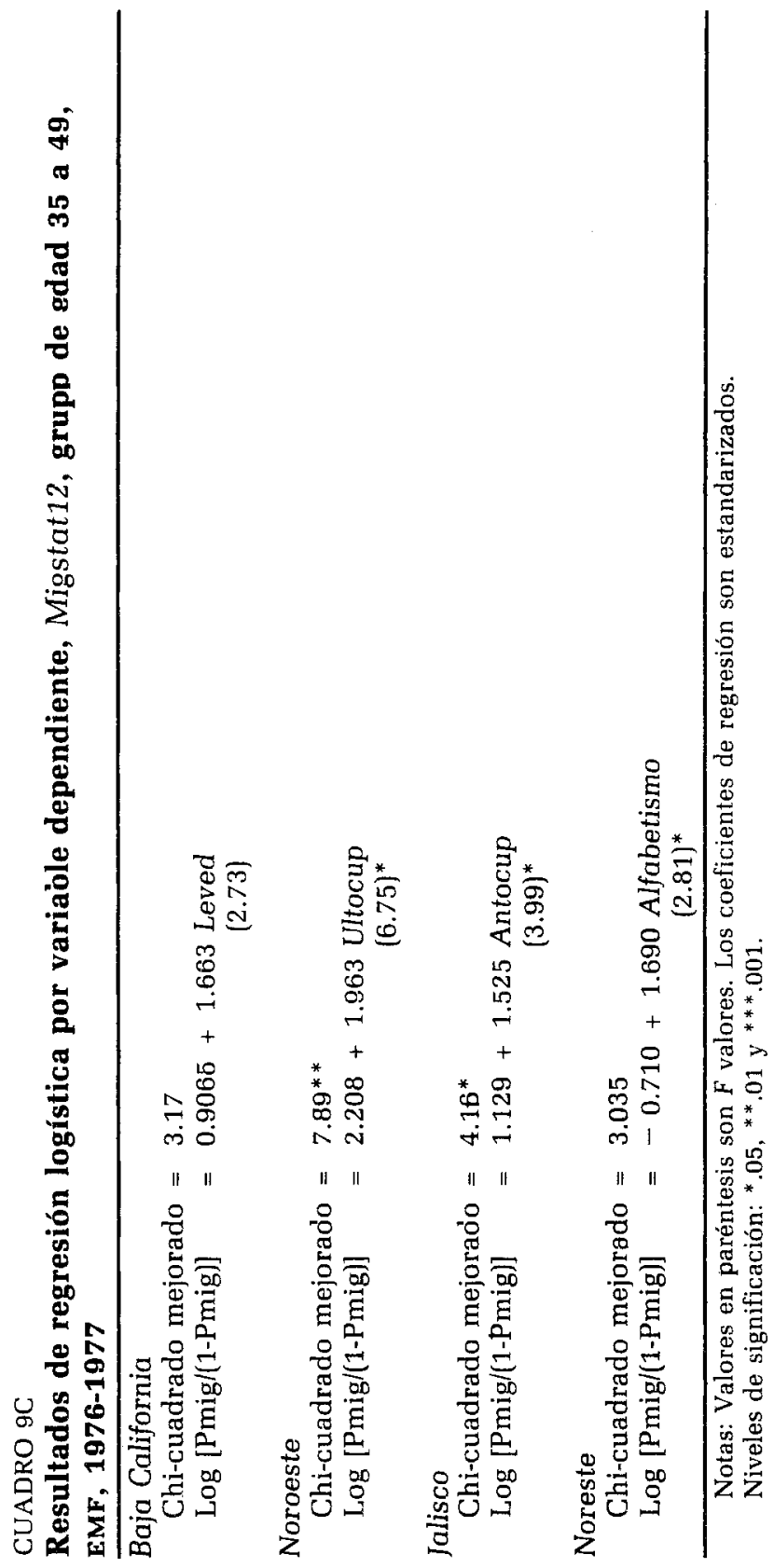


noreste, categoría 15 a 24 años, son probablemente sustitutos por el efecto positivo de edad; por ejemplo, los informantes más educados son los más jóvenes, ya que, los niveles educacionales mexicanos incrementaron grandemente en los años sesenta y setenta y la gente más joven ha tenido más corto plazo de mudarse. Hubo dos efectos positivos del alfabetismo, para la región noreste, edad 15 a 24 y para la región noroeste, edad 35 a 49 . Estos efectos son inexplicablemente opuestos a los efectos de Pleved. La discusión para el efecto negativo de Ninulnac ( -5 años) para la región noreste, edad 15 a 25 , la dejamos para una sección posterior sobre influencias de la fecundidad sobre la migración.

Efectos específicos de la edad para transiciones de mudanzas 1-2+

Tanto la transición de mudanza de 1-2 +, como los efectos específicos de la edad para las variables importantes Antocup y Ultocup están presentes en los cuatro casos, pero son más débiles que los efectos de conjunto por grupos de edad. Para cada región la especificidad de la edad varía. Para la región noroeste, el efecto Ultocup ocurre en las categorías 15 a 24 y 35 a 49 años; para Jalisco el efecto Antocup ocurre en la categoría 35 a 49; y para la región noreste, el efecto Antocup ocurre en el grupo de 15 a 24. Tamlugar y Pinbalugar son de importancia secundaria para Jalisco para las edades de 25 a 34 . Adicionalmente, Ninulnac ( -5 años) ejerce un efecto positivo en Baja California y un efecto negativo en la región noreste, categoría 25 a 34 .

En los análisis de las regresiones lineales y logísticas, hubo únicamente tres efectos significativos de fecundidad, todos aparecen en las regresiones logísticas. Existe un efecto positivo fuerte de Ninulnac ( -5 años) para Baja California, en transición de mudanza de 1-2 +, tanto para el agrupamiento total por edades como para la categoría 25 a 34 años. Esta variable mide fecundidad en la etapa posterior al Programa Bracero de 1971 a 1977. En este periodo, la tasa de inmigración a Baja California disminuyó. Una posible explicación errónea es que durante este periodo el ingreso por familia era superior y había una mayor probabilidad tanto de migrar como de tener más hijos. Sin embargo, se necesitaría una explicación detallada basada en investigación histórica sobre Baja California, que está más allá de los objetivos de este trabajo. Sin embargo, es importante hacer notar que no hay estudios que traten este tema para Baja California.

Hubo dos efectos negativos de Ninulnac ( -5 años) en el nores- 
te, para la categoría 15 a 24 años con la transición de mudanza 0-1 + y para la categoría 25 a 34 años con un estado transitorio 1-2 + . Consideramos que en específico para esta región, cohortes de edad y estados transitorios, fecundidad reducen la migración a través de un incremento en la estabilidad geográfica de la familia debido a los costos migratorios tanto económicos como emocionales.

\section{Discusión}

En este estudio se examinaron las determinantes de migración y sus interacciones para cuatro regiones en México. Comparando las cuatro regiones se obtienen las siguientes conclusiones:

1. En general la población es altamente migratoria ( 67 a $98 \%$ ).

2. El nivel de educación de la población es bajo y el nivel de analfabetas es alto, 9 a $18 \%$ en las personas mayores. bajar.

3. El matrimonio trae como resultado que la mujer deje de tra-

4. Las diferencias regionales se dan únicamente para algunas variables pero no para todas. Una diferencia consistenté y significativa es la mayor educación y mayor alfabetización en Baja California comparada con las otras tres regiones.

5. La población de Baja California es significativamente más migratoria y muestra incrementos de migración significativos para los cohortes con edades más avanzadas que en otras regiones. Las otras regiones tienen tasas de migración más bajas y no son significativamente diferentes entre ellas. No existen diferencias notables entre los dos grupos de edad más avanzados.

Adicionalmente, las siguientes conclusiones generales se pueden establecer con respecto a las influencias socioeconómicas sobre la migración:

1. Se nota una sucesión de influencias socieconómicas conforme los estados transitorios de movilidad se alteran. Para la transición de mudanza 0-1 +, existe un efecto mayor del lugar de residencia durante la niñez y una interacción con la residencia actual; para la transición 1-2 + hay efectos de la ocupación de los informantes y para la transición 2-3 + encontramos efectos inconsistentes de edad y ocupación.

2. Baja California difiere sustancialmente de las otras tres regiones en cuanto a los efectos socioeconómicos dominantes.

3. No existen diferencias consistentes de edad en las influen- 
cias socioeconómicas por los movimientos transitorios. Las diferencias por edad encontradas son específicas para cada región.

4. Los resultados obtenidos tanto en la regresión lineal como en la regresión logística son consistentes. Ambos tipos de análisis tienen ventajas y desventajas dependiendo del factor migratorio de que se trate.

5. Los cinco patrones de dirección consistente que existen son: a) un efecto negativo de residencia urbana durante la niñez, b) una interacción positiva con la residencia actual, c) un efecto positivo de la presencia de una ocupación antes o después del matrimonio, d) un efecto positivo de la educación primaria o alfabetización a menos de que un efecto de edad esté presente y e) el efecto positivo de la edad.

6. Los efectos de fecundidad sobre migración son menores. Los únicos efectos significativos encontrados son positivos para Baja California en todas las categorías de edades agrupadas y el grupo 25 a 34 y un efecto negativo fue para la región noreste, edades 15 a 34. Estos efectos menores corresponden a los resultados anteriormente encontrados sobre la carencia de efectos de migración sobre fecundidad.

7. Para el flujo migratorio históricamente más importante hacia Baja California, no hay evidencia de similitudes en las determinantes migratorias entre las regiones de emigrantes o entre la región de inmigrantes de Baja California y la región comparativa del noreste.

En resumen, este estudio revela las diferencias sustanciales de las influencias socioeconómicas sobre la migración de acuerdo con la edad, región y número anterior de mudanza. Estos resultados reflejan, en parte, diferentes flujos migratorios, así como también características migratorias contrastantes y las posibles variantes de las motivaciones. Es aparente la fuerte variación regional y corresponde a los resultados similares encontrados por Rollwagen (1971), aunque sus datos son a nivel de un solo pueblo y para los años sesenta. Las variantes generales de los resultados por región son también apoyados por los resultados encontrados por Muñoz et al., para la ciudad de México y por Browning y Feindt para Monterrey. Por ejemplo, Muñoz et al., reportan que tanto hombres como mujeres migrantes de pequeñas y medianas áreas urbanas tuvieron una alfabetización y nivel educativo significativamente más altos que los migrantes de zonas rurales o grandes zonas urbanas. Este resultado contrasta con los resultados encontrados en Monterrey de un incremento graduado del nivel educativo de migrantes, conforme a nivel económico y presumiblemente urbanización del lugar de origen. 
Las diferencias mayores encontradas son en las influencias socioeconómicas por transiciones de mudanzas para todas las regiones excepto Baja California con una mudanza inicial caracterizada por efectos residenciales, la segunda mudanza y posteriores por efectos ocupacionales y la tercera y posteriores mudanzas por efectos contrastantes educacionales y de edad. Esta sucesión de influencias socioeconómicas, de acuerdo a como se van haciendo las mudanzas, implican que cada mudanza requiere un cambio de motivaciones y susceptibilidades para mudanzas posteriores. La mudanza inicial al parecer está basada en fuerzas mayores de urbanización, mientras que la segunda mudanza quizás se debe a influencias más activas de búsqueda de trabajo. Las fuerzas que influyen tanto en la tercera mudanza como posteriores son menos claras, debido a los impactos tanto de la edad como de la ocupación. Nunca antes se había reportado en estudios cuantitativos de migración interna en México el cambio en la sucesión de influencias sobre las mudanzas, aunque estudios de campo indican diferentes motivaciones de los migrantes indocumentados de México a Estados Unidos y de regreso a México. Uno de los problemas en el examen de mudanzas sucesivas es la carencia de encuestas en donde se hayan incluido preguntas sobre la historia de la migración.

Los efectos socioeconómicos en las diferentes edades son inconsistentes. Son específicos a la historia de la migración de una categoría de edad en particular en una región específica. Eventualmente en el rango total de edades 15 a 49 , se nota un efecto pero el rango de edades para el efecto es inconsistente. Las diferencias en las categorías por edad para una región en particular se tiene que analizar eventualmente de acuerdo con la historia de los flujos migratorios y eventos sociales para tener una explicación completa. Este tipo de análisis está fuera del objetivo de este estudio.

La carencia de efectos sistemáticos parece contraria a la influencia dominante encontrada para la edad en los estudios de regresión de migración interna en México hechos anteriormente (Greenwood y Ladman, 1978, 1981; Fukurai et al., 1987a). Aunque estos estudios se basaron en datos de censos agrupados por unidades geográficas. La agregación quizás forzó una reducción de la importancia de algunos efectos (por ejemplo, ocupación, lugar de residencia).

Adicionalmente, la variable dependiente en estos estudios fue número de migrantes, lo cual difiere de número de mudanzas y transiciones específicas de mudanzas.

Aunque existe variación considerable en los resultados de 
nuestro estudio, según el número de mudanzas anteriores, región y edad, algunas influencias socioeconómicas son consistentes relativamente. Los patrones son los siguientes:

1. Residencia durante la niñez [del informante o compañero(a)] en zonas urbanas tiene un efecto negativo sobre la migración.

2. La residencia actual está relacionada positivamente con la migración.

3. La presencia de una ocupación para las mujeres informantes antes o después del matrimonio tiene un efecto positivo sobre la migración.

4. Educación primaria y alfabetización [del informante o compañero(a)] generalmente tienen un efecto positivo sobre la migración.

5. La edad tiene un efecto positivo sobre la migración.

6. Si el efecto de educación está presente junto con el efecto positivo de edad, la educación ejerce un efecto negativo sobre la migración.

Tanto el primero como el segundo patrón son el resultado de grandes flujos migratorios consistentes de zonas rurales a zonas urbanas, lo cual fue una de las dos causas más importantes de la urbanización en México en los últimos cincuenta años. Los patrones tercero y cuarto corresponden más cercanamente a los resultados encontrados por Browning para Monterrey. Estos patrones quizás reflejan en general las fuerzas sociales en las regiones norte y noreste de México. En futuras investigaciones sería interesante determinar si los patrones tres y cuatro persisten en regiones marcadamente diferentes a las estudiadas como son las regiones del sur, tanto del Golfo como del Pacífico mexicano. El quinto patrón es el resultado directo de la edad en el incremento potencial de migración. El sexto patrón está asociado con el quinto ya que indirectamente refleja los efectos de la edad.

Las débiles interacciones que se notaron entre fecundidad y la migración tanto en este estudio como en un estudio anterior hecho por Pick et al. (1988) quizás sea una característica de la parte norte y oeste de México y no de otras regiones. Son necesarias investigaciones en regiones totalmente diferentes en México para que se clarifiquen los patrones nacionales y regionales de las interacciones entre fecundidad y migración. 


\section{Bibliografía}

Bieber, Stephen L. (1988), "Multiple Regression and Its Alterntives", The Social Science Journal, vol. 25, mím. 1, pp. 1-19.

Bogue, Donald (1985), The Population of the United States, The Free Press. New York.

Brambila Paz, Carlos (1982), "Migration and Family Formation in Contemporary Mexico", Working paper, University of Chicago, Illinois.

Brown, Lawrence A. y Andrew R. Goetz (1987), "Development-Related Contextual Effects and Individual Attributes in Third World Migration Processes: A Venezuelan Example", Demography, vol. 24, núm. 4, pp. 497-516

y John Paul Jones III (1985), "Spatial Variation in Migration Processes and Development: A Costa Rican Example of Conventional Modelling Augmented by the Expansion Method", Demography, vol. 22, núm. 3, pp. 327-352.

Browning, Harley L. y Waltraut Feindt (1970), "The Social and Economic Context of Migration to Monterrey, Mexico", en F. F. Rubinowitz y F.M. Trueblood (comps.) Latin American Urban Research, vol. 1, Sage Publications, Beverly Hills, California, pp. 45-70.

- y Waltraut Feindt (1969), "Selectivity of Migrants to a Metropolis in a Developing Country: A Mexican Case Study, Demography, vol. 6, núm. 4, pp. 347-357.

y Waltraut Feindt (1968), "Diferencias Entre la Población Nativa y la Migrante en-Monterrey", Demografía y Economía, vol. 2, pp. 183204.

Butler, Edgar W., James B. Pick, Hiroshi Fukurai y Shas Pavgi (1987), "Migration to Baja California, 1900-1980", Research Paper, núm. 26, Center for Inter-American and Border Studies, University of Texas at El Paso.

Cantú Gutiérrez, Juan José (1986), "La migración internacional y sus efectos en la frontera norte: retos para la acción pública", artículo presentado en la Conferencia sobre Municipios Fronterizos del Norte, El Colegio de la Frontera Norte, Tijuana, B.C., Abril 23-26.

Cole, William E. y Richard D. Sanders (1983), "Interstate Migration in Mexico: Variations of the Todaro Theme", Journal of Development Economics, vol. 12, |núm.' 3, pp. 341-354.

Dixon, Wilfred et al., comps. (1985), BMDP Statistical Software, University of California Press, Berkeley.

Edlefsen, L.E. y B.S. Lee (1983), "The Effect of Rural/Urban Migration on Migrants Fertility: Evidence from Mexico", ponencia presentada en la Annual Meeting of the Population Association of America, Pittsburgh, Pennsylvania, $12 \mathrm{pp}$.

Fienberg, Stephen E. (1980), The Analysis of Cross-Classified Categorical Data, The MIT Press, Cambridge, Massachusetts.

Fukurai, Hiroshi, James B. Pick, Edgar W. Butler y Swapan Nag (1987a), "An Analysis of the Interstate Migration in Mexico", Mexican Studies/Estudios Mexicanos, vol. 3, núm. 3, pp. 365-395. 
y James B. Pick, Edgar W. Butler (1987b), "Interstate Migration in Mexico, 1980: A Spatial Analysis", Sociology and Social Research, vol. 71 , núm. 4, pp. 312-322.

Greenwood, Michael J., Jerry R. Ladman y Barry S. Siegel (1981), "LongTerm Trends in Migratory Behavior in a Developing Country: The Case of México", Demography, vol. 18, núm. 3, pp. 369-388

y Jerry R. Ladman (1978), "An Economic Analysis of Migration in Mexico", Annals of Regional Science, vol. 12, núm. 2, pp. 16-31. y Jerry R. Ladman (1977), "Economía de la movilidad geográfica de la mano de obra en México", Demografía y Economía, vol. 11, núm. 2, pp. 155-166.

Jones, Richard C. (1984), "Macro-Patterns of Undocumented Migration Between Mexico and the U.S.", en Richard C. Jones (comp.), Patterns of Undocumented Migration, Littlefield, Adams, and Company, Totawa, New Jersey.

Muñoz Izquierdo, Carlos (1973), "Evaluación del desarrollo educativo en México (1958-70) y factores que lo han determinado", Revista del Centro de Estudios Educativos 3(3).

Muñoz Humberto, Orlandina de Oliveira y Claudio Stern (1973), "Migración interna y movilidad ocupacional en la ciudad de México", Demografía y Economía, vol. 2, pp. 135-148.

Orlandina de Oliveira y Claudio Stern (1972), "Migración y marginalidad ocupacional en la ciudad de México", en: El Perfil de México en 1980, vol. 3, México, Siglo XXI Editores.

Ordorica, Manuel y Joseph E. Potter (1981), "Evaluation of the Mexico Fertility Survey, 1976-77", Scientific Report, núm. 21, World Fertility Survey, Voorburg, The Gague, Netherlands, $29 \mathrm{pp}$.

Pick, James B., Edgar W. Butler y Suhas Pavqui (1988), "Socioeconomic Determinants of Fertility, Selected Mexican Regions, 1976-77”, Social Biology, vol. 35, núm. 1-2, 137-157.

Population Reference Bureau (1988), "1988 World Population Data Sheet", Population Reference Bureau, Inc., Washington, D.C.

SPP (1984), X Censo General de Población y Vivienda, 1980, varios volúmenes. Secretaría de Programación y Presupuesto, México.

(1979a), Encuesta Mexicana de Fecundidad, vol. I, Primer Informe Nacional, Secretaría de Programación y Presupuesto, México.

(1979b), Encuesta Mexicana de Fecundidad, vol. II, Primer Informe Nacional, Secretaría de Programación y Presupuesto, México.

(1978) Encuesta Mexicana de Fecundidad, Informe Metodológico, Secretaría de Programación y Presupuesto, México.

(1975), IX Censo General de Población y Vivienda, 1970, varios volúmenes. Secretaría de Programación y Presupuesto, México.

Scott, Ian (1982), Urban and Spatial Development in Mexico, The Johns Hopkins University Press, Baltimore.

Unikel, Luis (1977), "Urbanization in Mexico: Process, Implications, Policies, and Prospects", en Sidney Goldstein and David Sly (comps.), Patterns of Urbanization: Comparative Country Studies, Ordina Editions, Dolhain, Belgium, pp. 465-568. 
, Crescencio Ruiz Ch. y Omar Lazcano (1973), "Factores de rechazo en la migración rural en México, 1950-60", Demografía y Economía, vol. 12, pp. 24-57.

Vásquez, Rodolfo (1984), “Algunos aspectos cuantitativos sobre la relación entre la emigración internacional y la migración interna de mexicanos", Estudios Fronterizos, vol. 1, núm. 3, pp. 113-132.

Whetten, Nathan y Robert G. Burnight (1956), "Internal Migration in Mexico", Rural Sociology vol. 21 (junio), pp. 140-151.

Winnie, William W. (1981), "Componentes del crecimiento y redistribución de la población mexicana: implicaciones de los resultados preliminares del Censo de 1980", Demografía y Economía, vol. 15, núm. 3, pp. 359-376. 
